\title{
On scaling limits and Brownian interlacements*
}

\section{Alain-Sol Sznitman}

\begin{abstract}
We consider continuous time interlacements on $\mathbb{Z}^{d}, d \geq 3$, and investigate the scaling limit of their occupation times. In a suitable regime, referred to as the constant intensity regime, this brings Brownian interlacements on $\mathbb{R}^{d}$ into play, whereas in the high intensity regime the Gaussian free field shows up instead. We also investigate the scaling limit of the isomorphism theorem of [40]. As a by-product, when $d=3$, we obtain an isomorphism theorem for Brownian interlacements.
\end{abstract}

Keywords: Bownian interlacements, scaling limits, isomorphism theorems.

Mathematical subject classification: 60F05, 60J27, 60G60, 60J65.

\section{Introduction}

Informally, random interlacements provide a model for the local structure left at appropriately chosen time scales by random walks on large recurrent graphs, which are locally transient. Their vacant set has non-trivial percolative properties, see for instance [9], [31], [38], [43], which are useful in the study of certain disconnection and fragmentation problems, see [6], [7], [37], [44]. Their connectivity properties have been actively investigated, see [5], [20], [28], [29], [30]. Random interlacements have further been helpful in some questions of cover times, see [1], [2]. They have also been linked to Poisson gases of Markovian loops, see [23], [41]. Recently, connections between random interlacements and Gaussian free fields have emerged, which underline the important role of occupation times of random interlacements, see [40].

In this article, we investigate the scaling limit of the field of occupation times of continuous time interlacements on $\mathbb{Z}^{d}, d \geq 3$. In the constant intensity regime, this brings into play the Brownian interlacements on $\mathbb{R}^{d}, d \geq 3$. In the high intensity regime, the massless Gaussian free field shows up instead. Further,

Received 21 September 2012.

*This research was supported in part by the grant ERC-2009-AdG 245728-RWPERCRI. 
we investigate the scaling limit of the isomorphism theorem derived in [40]. Dimension three plays a special role, and when $d=3$, we obtain as a limit an identity in law relating the occupation-time measure of Brownian interlacements on $\mathbb{R}^{3}$ to the massless Gaussian free field, somewhat in the spirit of [22], [23], in the context of Poisson gases of Brownian loops at half-integer levels.

We now discuss our results in more detail. We consider continuous time random interlacements on $\mathbb{Z}^{d}, d \geq 3$. In essence, this is a Poisson point process on a certain state space consisting of doubly infinite $\mathbb{Z}^{d}$-valued trajectories marked by their duration at each step, modulo time-shift. A non-negative parameter $u$ plays the role of a multiplicative factor of the intensity measure of this Poisson point process, which is defined on a suitable canonical space, see [39], denoted here by $(\bar{\Omega}, \overline{\mathcal{A}}, \overline{\mathbb{P}})$. The field of occupation times of random interlacements at level $u$ is denoted by $L_{x, u}(\omega)$, for $x \in \mathbb{Z}^{d}, u \geq 0, \omega \in \bar{\Omega}$. It records the total duration spent at $x$ by the trajectories modulo time-shift with label at most $u$ in the cloud $\omega$, see [39].

We investigate the scaling limit for the field of occupation times of random interlacements on $\mathbb{Z}^{d}, d \geq 3$, and introduce the random measure on $\mathbb{R}^{d}$

$$
\mathcal{L}^{N}=\frac{1}{d N^{2}} \sum_{x \in \mathbb{Z}^{d}} L_{x, u_{N}} \delta_{\frac{x}{N}}, \quad N \geq 1,
$$

where $\left(u_{N}\right)_{N \geq 1}$ is a suitably chosen sequence of positive levels. In the constant intensity regime, that is when

$$
u_{N}=d \alpha N^{2-d}, \text { with } \alpha>0
$$

(in this case the intensity measure $\overline{\mathbb{E}}\left[\mathcal{L}^{N}\right]$ of $\mathcal{L}^{N}$ converges vaguely to $\alpha d y$ as $N$ goes to infinity), we show in Theorem 3.2 that

$$
\mathcal{L}^{N} \text { converges in distribution to } \mathcal{L}_{\alpha} \text {, as } N \rightarrow \infty,
$$

where $\mathcal{L}_{\alpha}$ stands for the occupation-time measure of Brownian interlacements at level $\alpha$, see (2.37). We construct Brownian interlacements in Section 2 using a similar strategy as in [38] or [42]. This is technically somewhat more involved in the present context, and the key identity is encapsulated in Lemma 2.1. The random measure $\mathcal{L}_{\alpha}$ is supported by the random closed set $\mathcal{I}_{0}^{\alpha}$, the Brownian fabric at level $\alpha$, which is the union of the traces in $\mathbb{R}^{d}$ of the doubly infinite trajectories modulo time-shift with label at most $\alpha$ in the Poisson cloud defining the Brownian interlacements, see (2.30). This random closed subset of $\mathbb{R}^{d}$ is a.s. connected when $d=3$, but a.s. disconnected when $d \geq 4$, see Proposition 2.5. 
On the other hand in the high intensity regime, that is when

$$
N^{d-2} u_{N} \rightarrow \infty,
$$

we show in Theorem 3.3 and Corollary 3.5 that

$$
\widehat{\mathcal{L}}^{N}=\sqrt{\frac{d}{2 N^{2-d} u_{N}}}\left(\mathcal{L}^{N}-\overline{\mathbb{E}}\left[\mathcal{L}^{N}\right]\right) \begin{aligned}
& \text { converges in distribution to the } \\
& \text { massless Gaussian free field } \Phi \text { on } \mathbb{R}^{d},
\end{aligned}
$$

which is the canonical generalized random field on $S^{\prime}\left(\mathbb{R}^{d}\right)$, the space of tempered distributions on $\mathbb{R}^{d}$, such that for any $V$ in the Schwartz space $S\left(\mathbb{R}^{d}\right)$, the pairing $\langle\Phi, V\rangle$ is a centered Gaussian variable with variance $\int V(y) G(y-$ $\left.y^{\prime}\right) V\left(y^{\prime}\right) d y d y^{\prime}$, with $G(\cdot)$ the Green function of Brownian motion on $\mathbb{R}^{d}$, $d \geq 3$, see (1.3).

The low intensity regime, when $N^{d-2} u_{N} \rightarrow 0$, leads to a null limit for $\mathcal{L}^{N}$ in (0.1), and will not be further discussed in the present work.

From the isomorphism theorem for random interlacements, see [40], one knows that when $\left(\varphi_{x}\right)_{x \in \mathbb{Z}^{d}}$ is a discrete massless Gaussian free field, i.e. a centered Gaussian field with covariance $E\left[\varphi_{x} \varphi_{x^{\prime}}\right]=g\left(x-x^{\prime}\right)$, with $g(\cdot)$ the Green function of simple random walk on $\mathbb{Z}^{d}$, see (1.1), independent from $\left(L_{x, u}\right)_{x \in \mathbb{Z}^{d}}$, one has for any $u \geq 0$ the distributional identity:

$$
\left(\frac{1}{2} \varphi_{x}^{2}+L_{x, u}\right)_{x \in \mathbb{Z}^{d}} \stackrel{\text { law }}{=}\left(\frac{1}{2}\left(\varphi_{x}+\sqrt{2 u}\right)^{2}\right)_{x \in \mathbb{Z}^{d}} .
$$

We explain in Section 4 how one quickly recovers (0.5) from this identity in the regime where, in essence, as $N \rightarrow \infty$, the variance of $\sum_{|x| \leq N} \varphi_{x}^{2}$ is negligible compared to that of $\sqrt{u}_{N} \sum_{|x| \leq N} \varphi_{x}$. This corresponds to the full range (0.4) when $d=3$, but only the partial range of (0.4) where $u_{N} N^{2} \log N \rightarrow \infty$, when $d=4$, and $u_{N} N^{2} \rightarrow \infty$, when $d \geq 5$, see Remark 4.2. This fact singles out the special role of dimension 3 , and motivates the investigation when $d=3$ of the scaling limit of (0.6) (with adequate counter terms) in the constant intensity regime (0.2). Indeed, when $d=3$, Theorem 5.1 roughly states that for any $\alpha \geq 0$ (denoting Wick products by ::, see Section 5 )

$$
\frac{1}{d N^{2}} \sum_{x \in \mathbb{Z}^{d}}:\left(\varphi_{x}+\sqrt{2 u_{N}}\right)^{2}: \delta_{\frac{x}{N}} \text { converges in distribution to }:(\Phi+\sqrt{2 \alpha})^{2}:,
$$

with $u_{N}$ as in (0.2), and the last term defined by regularization of the massless Gaussian free field $\Phi$ on $\mathbb{R}^{3}$ (crucially using the fact that the Green function is 
locally square integrable when $d=3$ ), see the beginning of Section 5 . As a scaling limit of (0.6) we obtain the identity in law on $S^{\prime}\left(\mathbb{R}^{3}\right)$, for $\alpha \geq 0$ :

$$
\frac{1}{2}: \Phi^{2}:+\mathcal{L}_{\alpha}=\frac{1}{2}:(\Phi+\sqrt{2 \alpha})^{2}:,
$$

where $\mathcal{L}_{\alpha}$ is independent of $\Phi$ and denotes, as above, the occupation-time measure of Brownian interlacements on $\mathbb{R}^{3}$ at level $\alpha$, see Corollary 5.3. This identity in law has a similar spirit to some of the results in [22], [23] in the context of Poisson gases of Brownian loops at half-integer levels, see Remark 5.4.

Let us describe how the article is organized. In Section 1 we collect some useful facts concerning Green functions, occupation times of random interlacements on $\mathbb{Z}^{d}$, and discrete as well as continuous free fields. Section 2 is of independent interest. It constructs the Brownian interlacements on $\mathbb{R}^{d}, d \geq 3$, and derives some of their key properties, see Propositions 2.4, 2.5, 2.6. In Section 3, we investigate the limit of $\mathcal{L}^{N}$, respectively $\widehat{\mathcal{L}}^{N}$, in the constant intensity, respectively, high intensity regime. The main results appear in Theorems 3.2, 3.3 and Corollary 3.5. In the short Section 4 we use the isomorphism theorem $(0.6)$ as a mean to recover $(0.5)$. Section 5 focuses on the three-dimensional situation. The scaling limit of (0.6) (with adequate counter terms) is investigated in the constant intensity regime (0.2). The main results appear in Theorem 5.1 and Corollary 5.3.

Finally, let us explain our convention concerning constants. We denote by $c, c^{\prime}, \tilde{c}, \bar{c}$ positive constants changing from place to place, which simply depend on $d$. Numbered constants $c_{0}, c_{1}, \ldots$ refer to the value corresponding to their first appearance in the text. Dependence of constants on additional parameters appears in the notation.

\section{Some useful facts}

In this section, we introduce additional notation, and collect some useful facts concerning Green functions, occupation times of random interlacements on $\mathbb{Z}^{d}$, $d \geq 3$, and massless Gaussian free fields on $\mathbb{Z}^{d}$ and $\mathbb{R}^{d}, d \geq 3$.

We write $|\cdot|$, respectively $|\cdot|_{\infty}$, for the Euclidean, respectively, the supremum norm on $\mathbb{R}^{d}$. Throughout, we tacitly assume $d \geq 3$. We let $B(y, r), y \in \mathbb{R}^{d}$, $r \geq 0$, stand for the closed Euclidean ball with center $y$ and radius $r$.

We denote by $g(\cdot, \cdot)$ the Green function of simple random walk on $\mathbb{Z}^{d}$, that is, for $x, x^{\prime} \in \mathbb{Z}^{d}, g\left(x, x^{\prime}\right)$ is the expected time spent at $x^{\prime}$ for the discrete time, simple random walk starting at $x$. The function $g(\cdot, \cdot)$ is symmetric, and due to translation invariance, one has for $x, x^{\prime}$ in $\mathbb{Z}^{d}$

$$
g\left(x, x^{\prime}\right)=g\left(x-x^{\prime}\right)=g\left(x^{\prime}-x\right), \text { where } g(\cdot)=g(\cdot, 0) .
$$


One knows that $g(\cdot) \leq g(0)$ and that when $x$ tends to infinity, cf. [21], p. 31,

$$
\begin{aligned}
& g(x) \sim d G(x), \text { where } \\
& G(y)=\frac{1}{2 \pi^{d / 2}} \Gamma\left(\frac{d}{2}-1\right)|y|^{2-d}, \text { for } y \in \mathbb{R}^{d},
\end{aligned}
$$

and " " in (1.2) means that the ratio of the two members tends to 1 as $x$ goes to infinity. Writing $G\left(y, y^{\prime}\right)=G\left(y-y^{\prime}\right)=G\left(y^{\prime}-y\right)$, one knows, see [10], p. 32, that the $(0, \infty]$-valued function $G(\cdot, \cdot)$ is the Green function of Brownian motion on $\mathbb{R}^{d}$.

For $N \geq 1$, we denote by $\mathbb{L}_{N}$ the lattice in $\mathbb{R}^{d}$ :

$$
\mathbb{L}_{N}=\frac{1}{N} \mathbb{Z}^{d}
$$

For functions $f, h$ on $\mathbb{L}_{N}$ such that $\sum_{y \in \mathbb{L}_{N}}|f(y) h(y)|<\infty$, we write

$$
\langle f, h\rangle_{\mathbb{L}_{N}}=\frac{1}{N^{d}} \sum_{y \in \mathbb{L}_{N}} f(y) h(y) .
$$

We rescale the Green function $g(\cdot, \cdot)$ and define

$$
g_{N}\left(y, y^{\prime}\right)=\frac{1}{d} N^{d-2} g\left(N y, N y^{\prime}\right), \text { for } y, y^{\prime} \in \mathbb{L}_{N},
$$

as well as $g_{N}(\cdot)=g_{N}(\cdot, 0)$, so that $g_{N}\left(y, y^{\prime}\right)=g_{N}\left(y-y^{\prime}\right)=g_{N}\left(y^{\prime}-y\right)$. By (1.2), (1.3), we also see that

$$
\lim _{N} \sup _{|y| \geq \gamma}\left|g_{N}(y)-G(y)\right|=0, \text { for every } \gamma>0 .
$$

We introduce the linear operator

$$
G_{N} f(y)=\frac{1}{N^{d}} \sum_{y^{\prime} \in \mathbb{L}_{N}} g_{N}\left(y, y^{\prime}\right) f\left(y^{\prime}\right), \quad y \in \mathbb{L}_{N},
$$

which is well-defined when the function $f: \mathbb{L}_{N} \rightarrow \mathbb{R}$ is such that

$$
\sum_{y \in \mathbb{L}_{N}} g_{N}\left(y, y^{\prime}\right)\left|f\left(y^{\prime}\right)\right|<\infty
$$

for some (and hence all) $y$ in $\mathbb{L}_{N}$, in particular, when $f$ vanishes outside a finite set. 
Similarly, when $f, h$ are measurable functions on $\mathbb{R}^{d}$ such that $|f h|$ is integrable, we write

$$
\langle f, h\rangle=\int_{\mathbb{R}^{d}} f(y) h(y) d y .
$$

We also introduce the linear operator

$$
G f(y)=\int_{\mathbb{R}^{d}} G\left(y, y^{\prime}\right) f\left(y^{\prime}\right) d y^{\prime}, \text { for } y \in \mathbb{R}^{d},
$$

which is well-defined when the measurable function $f$ on $\mathbb{R}^{d}$ satisfies

$$
\int G\left(y, y^{\prime}\right)\left|f\left(y^{\prime}\right)\right| d y^{\prime}<\infty
$$

for all $y \in \mathbb{R}^{d}$, in particular when $f$ is bounded measurable and vanishes outside a bounded set.

We now recall an identity for the Laplace transform of the field of occupation times $\left(L_{x, u}\right)_{x \in \mathbb{Z}^{d}}$ of continuous time random interlacements on $\mathbb{Z}^{d}$ at level $u \geq 0$. When $V: \mathbb{L}_{N} \rightarrow \mathbb{R}$ has finite support, the operator $G_{N} V$, which is the composition of the multiplication by $V$ with the operator $G_{N}$ in (1.8), sends bounded functions on $\mathbb{L}_{N}$ into bounded functions on $\mathbb{L}_{N}$. We write $\left\|G_{N} V\right\|_{L^{\infty} \rightarrow L^{\infty}}$ for the corresponding operator norm (the space of bounded functions on $\mathbb{L}_{N}$ being endowed with the sup-norm). One knows from Theorem 2.1 of [39] that when $V: \mathbb{L}_{N} \rightarrow \mathbb{R}$ has finite support and $\left\|G_{N}|V|\right\|_{L^{\infty} \rightarrow L^{\infty}}<1$, then for $u \geq 0$,

$$
\begin{aligned}
& \overline{\mathbb{E}}\left[\exp \left\{\sum_{x \in \mathbb{Z}^{d}} V\left(\frac{x}{N}\right) \frac{1}{d N^{2}} L_{x, u}\right\}\right] \\
= & \exp \left\{\frac{u}{d} N^{d-2}\left\langle V,\left(I-G_{N} V\right)^{-1} 1\right\rangle_{\mathbb{L}_{N}}\right\} .
\end{aligned}
$$

Note that when $V$ vanishes outside a single point one readily finds by differentiation that:

$$
\overline{\mathbb{E}}\left[L_{x, u}\right]=u \text {, for } x \in \mathbb{Z}^{d} \text { and } u \geq 0 .
$$

We then turn to the discussion of Gaussian free fields. Recall that we tacitly assume $d \geq 3$. We begin with the discrete case. We endow $\mathbb{R}^{\mathbb{Z}^{d}}$ with the product $\sigma$-algebra, and denote by $\left(\varphi_{x}\right)_{x \in \mathbb{Z}^{d}}$ the canonical coordinates. The canonical law $P^{g}$ of the massless Gaussian free field on $\mathbb{Z}^{d}$ is characterized by the fact that

under $P^{g},\left(\varphi_{x}\right)_{x \in \mathbb{Z}^{d}}$ is a centered Gaussian field with covariance

$$
E^{P^{g}}\left[\varphi_{x} \varphi_{x^{\prime}}\right]=g\left(x, x^{\prime}\right) \text {, for } x, x^{\prime} \in \mathbb{Z}^{d} \text {. }
$$


In the continuous case, we consider the space $S^{\prime}\left(\mathbb{R}^{d}\right)$ of tempered distributions on $\mathbb{R}^{d}$. We endow $S^{\prime}\left(\mathbb{R}^{d}\right)$ with the cylindrical $\sigma$-algebra generated by the canonical pairings with functions of $S\left(\mathbb{R}^{d}\right)$. We write $\Phi$ for the canonical generalized random field on $S^{\prime}\left(\mathbb{R}^{d}\right)$ (i.e. the identity map). The symmetric bilinear form on $S\left(\mathbb{R}^{d}\right) \times S\left(\mathbb{R}^{d}\right)$ defined by

$$
E(V, W)=\int V(y) G\left(y-y^{\prime}\right) W\left(y^{\prime}\right) d y d y^{\prime}(=\langle V, G W\rangle=\langle G V, W\rangle)
$$

is positive definite (see for instance [36], p. 75). It satisfies the bound

$$
|E(V, W)| \leq c\|V\|_{L^{1}\left(\mathbb{R}^{d}\right)}\left(\|W\|_{L^{\infty}\left(\mathbb{R}^{d}\right)}+\|W\|_{L^{1}\left(\mathbb{R}^{d}\right)}\right), \text { for } V, W \in S\left(\mathbb{R}^{d}\right),
$$

and hence is continuous (we endow $S\left(\mathbb{R}^{d}\right)$ with its usual Fréchet topology, see [17], p. 6-8). By Minlos' Theorem, see Theorem 2.3, p. 12 of [34], or Theorem 2.4 .1 , p. 28 of [17], there exists a unique probability measure $P^{G}$ on $S^{\prime}\left(\mathbb{R}^{d}\right)$ such that

$$
\text { under } P^{G} \text {, for each } V \in S\left(\mathbb{R}^{d}\right),\langle\Phi, V\rangle
$$

is a centered Gaussian variable with variance $E(V, V)$.

The law $P^{G}$ describes the massless Gaussian free field on $\mathbb{R}^{d}$, see Chapter $6 \S 2$ of [15].

\section{Brownian interlacements}

In this section we construct the Brownian interlacements on $\mathbb{R}^{d}, d \geq 3$, introduce their occupation-time measure, and discuss some key properties of these objects. We follow a similar approach as in [38], [42]. However, the continuous set-up is technically more challenging. An important step is the construction of the intensity measure of our basic point process on a space of doubly infinite trajectories modulo time-shift. We mainly rely on a crucial compatibility property of the measure expressed in "local charts", see Lemma 2.1 (cf. Theorem 1.1 of [38] and Theorem 2.1 of [42]), rather than on an approach based on projective limits, see [45], [32], following the outline of [16].

We first need to introduce notation. We recall that we tacitly assume $d \geq 3$. We denote by $W_{+}$the subspace of $C\left(\mathbb{R}_{+}, \mathbb{R}^{d}\right)$ of continuous $\mathbb{R}^{d}$-valued trajectories tending to infinity at infinite times, and by $W$ the subspace of $C\left(\mathbb{R}, \mathbb{R}^{d}\right)$ consisting of continuous bilateral trajectories from $\mathbb{R}$ into $\mathbb{R}^{d}$, which tend to infinity at plus and minus infinite times. We write $X_{t}, t \geq 0$, and $X_{t}, t \in \mathbb{R}$, for the respective canonical processes, and denote by $\theta_{t}, t \geq 0$, and $\theta_{t}, t \in \mathbb{R}$, 
the respective canonical shifts. The spaces $W_{+}$and $W$ are endowed with the respective $\sigma$-algebras $\mathcal{W}_{+}$and $\mathcal{W}$ generated by the canonical processes. Given an open subset $U$ of $\mathbb{R}^{d}, w \in W_{+}$, we write $T_{U}(w)=\inf \left\{s \geq 0 ; X_{s}(w) \notin U\right\}$ for the exit time of $U$. When $F$ is a closed subset of $\mathbb{R}^{d}$, we write

$$
\left.H_{F}(w)=\inf \left\{s \geq 0 ; X_{s}(w) \in F\right)\right\}, \widetilde{H}_{F}(w)=\inf \left\{s>0 ; X_{s}(w) \in F\right\},
$$

for the respective entrance time and hitting time of $F$. When $w \in W$, we define $H_{F}(w)$ and $T_{U}(w)$ by similar formulas, simply replacing the condition $s \geq 0$, by $s \in \mathbb{R}$.

We then consider $W^{*}$ the set of equivalence classes of trajectories in $W$ modulo time-shift, i.e. $W^{*}=W / \sim$, where $w \sim w^{\prime}$, when $w(\cdot)=w^{\prime}(\cdot+t)$ for some $t \in \mathbb{R}$. We denote by $\pi^{*}$ the canonical map $W \rightarrow W^{*}$, and introduce the $\sigma$-algebra $\mathcal{W}^{*}=\left\{A \subseteq W^{*} ;\left(\pi^{*}\right)^{-1}(A) \in \mathcal{W}\right\}$, which is the largest $\sigma$-algebra on $W^{*}$ such that $(W, \mathcal{W}) \stackrel{\pi^{*}}{\longrightarrow}\left(W^{*}, W^{*}\right)$ is measurable. Incidentally, note that a random variable $Z$ on $W$ invariant under $\theta$ (i.e. $Z \circ \theta_{t}=Z$, for all $t$ in $\mathbb{R}$ ) determines a unique random variable $Z^{*}$ on $W^{*}$ such that $Z^{*} \circ \pi^{*}=Z$. Given a compact subset $K$ of $\mathbb{R}^{d}$, we write $W_{K}$ for the subset of trajectories of $W$ that enter $K$, and $W_{K}^{*}$ for its image under $\pi^{*}$.

Since $d \geq 3$, and Brownian motion on $\mathbb{R}^{d}$ is transient, we view $P_{y}$, the Wiener measure starting from $y \in \mathbb{R}^{d}$, as defined on $\left(W_{+}, \mathcal{W}_{+}\right)$, and write $E_{y}$ for the corresponding expectation. When $\rho$ is a finite measure on $\mathbb{R}^{d}$, we write $P_{\rho}$ for the Wiener measure with "initial distribution" $\rho$ and $E_{\rho}$ for the corresponding expectation.

When $B$ is a closed Euclidean ball of positive radius (as a shorthand in what follows, we will simply write that $B$ is a closed ball), and when $y \notin B$, we define $P_{y}^{B}[\cdot]=P_{y}\left[\cdot \mid H_{B}=\infty\right]$ to be the law of Brownian motion starting at $y$ conditioned never to enter $B$, and write $E_{y}^{B}$ for the corresponding expectation. When $y \in \partial B$ (the boundary of $B$ ), as $z$ in $\mathbb{R}^{d} \backslash B$ tends to $y$, the measures $P_{z}^{B}$ converge weakly (on $C\left(\mathbb{R}_{+}, \mathbb{R}^{d}\right)$ ) to a measure $P_{y}^{B}$ supported on $\left\{w \in W_{+} ; w(0)=y\right.$ and $w(t) \notin B$ for all $t>0\}$, which can be represented as the Brownian excursion measure in $\mathbb{R}^{d} \backslash B$ starting from $y$ conditioned on the event of finite positive mass that $\left\{\widetilde{H}_{B}=\infty\right\}$ (see Theorems 4.1 and 2.2 of [4], and also Theorem 3.1 of [8] for bounds on the exit time from small balls centered at $y$ under $P_{z}^{B}$ ). Using rotational invariance and the explicit nature of the conditioning, one can also in a more direct fashion establish the above mentioned facts using a skew product representation of the law $P_{z}^{B}$.

We write

$$
p_{t}\left(y, y^{\prime}\right)=\frac{1}{(2 \pi t)^{d / 2}} \exp \left\{-\frac{\left|y-y^{\prime}\right|^{2}}{2 t}\right\},
$$


with $t>0, y, y^{\prime} \in \mathbb{R}^{d}$, for the Brownian transition density, so that with similar notation as below (1.3)

$$
G\left(y, y^{\prime}\right)=\int_{0}^{\infty} p_{t}\left(y, y^{\prime}\right) d t, \text { for } y, y^{\prime} \in \mathbb{R}^{d} .
$$

Given a compact subset $K$ of $\mathbb{R}^{d}$, we denote by $e_{K}$ the equilibrium measure of $K$ (see Proposition 3.3, p. 58 of [36], or Theorem 1.10, p. 58 of [27]). This measure does not have atoms (see [36], p. 58, or [27], p. 197). It is supported by the boundary of $K$ (actually, the boundary of the unbounded component of $K^{c}$, see [27], p. 58). Its total mass is the capacity cap $(K)$ of $K$. The equilibrium measure $e_{K}$ is related to the time of the last visit of $K$ via (see Theorem 3.4, p. 60 of [36]):

$$
P_{y}\left[L_{K}>0, L_{K} \in d t, X_{L_{K}} \in d z\right]=p_{t}(y, z) e_{K}(d z) d t,
$$

where for $w \in W_{+}$we write $L_{K}(w)=\sup \{t>0 ; w(t) \in K\}$ (with the convention $\sup \phi=0$ ), for the time of last visit of $w$ to $K$.

For $B$ a closed ball (see above (2.1) for the terminology), we introduce the following (finite) measure on $W_{B}^{0}=\left\{w \in W ; H_{B}(w)=0\right\}\left(\subseteq W_{B}\right)$, the subset of $W$ of bilateral trajectories that enter $B$ at time 0 for the first time,

$$
\begin{aligned}
& Q_{B}\left[\left(X_{-t}\right)_{t \geq 0} \in A^{\prime}, X_{0} \in d y,\left(X_{t}\right)_{t \geq 0} \in A\right] \\
& =e_{B}(d y) P_{y}^{B}\left[A^{\prime}\right] P_{y}[A], \text { for } A, A^{\prime} \in \mathcal{W}_{+} .
\end{aligned}
$$

The next lemma states an important compatibility property of the above collection of finite measures. In essence, the measures $Q_{B}$ should be thought of as the expressions in the (natural) "local charts" of $W^{*}$ with respective domains $W_{B}^{0}\left(\subseteq W_{B}\right)$ of the intensity measure we are trying to construct (see also below (1.15) of [38]).

Lemma 2.1. Assume that $B, B^{\prime}$ are closed balls, and $B$ lies in the interior of $B^{\prime}$, then

$$
\theta_{H_{B}} \circ\left(1\left\{H_{B}<\infty\right\} Q_{B^{\prime}}\right)=Q_{B} .
$$

We postpone the proof of Lemma 2.1 for the time being, and explain how the construction of the intensity measure of our basic Poisson point process proceeds. We first observe that for $K$ compact subset of $\mathbb{R}^{d}$ we can unambiguously define

$$
Q_{K}=\theta_{H_{K}} \circ\left(1\left\{H_{K}<\infty\right\} Q_{B}\right) \text {, for any closed ball } B \supseteq K,
$$


and this definition coincides with (2.3) when $K$ is a closed ball. Indeed, when the closed balls $B_{1}$ and $B_{2}$ contain $K$, we can find a closed ball $B^{\prime}$ containing $B_{1} \cup B_{2}$ in its interior, so that by Lemma 2.1 we have

$$
\begin{aligned}
\theta_{H_{K}} \circ\left(1\left\{H_{K}<\infty\right\} Q_{B_{1}}\right) & =\theta_{H_{K}}\left(1\left\{H_{K}<\infty\right\} \theta_{H_{B_{1}}} \circ\left(1\left\{H_{B_{1}}<\infty\right\} Q_{B^{\prime}}\right)\right) \\
& =\theta_{H_{K}} \circ\left(\theta_{H_{B_{1}}}\left(1\left\{H_{K}<\infty\right\} Q_{B^{\prime}}\right)\right) \\
& =\theta_{H_{K}} \circ\left(1\left\{H_{K}<\infty\right\} Q_{B^{\prime}}\right),
\end{aligned}
$$

and a similar identity holds with $B_{2}$ in place of $B_{1}$. Hence (2.5) is unambiguous and agrees with (2.3) when $K$ is a closed ball.

We now come to the theorem constructing what will be in essence up to a nonnegative multiplicative factor the intensity measure of the Poisson point process defining the Brownian interlacements.

Theorem 2.2. There exists a unique $\sigma$-finite measure $v$ on $\left(W^{*}, W^{*}\right)$ such that for each compact subset $K$ of $\mathbb{R}^{d}$

$$
1_{W_{K}^{*}} v=\pi^{*} \circ Q_{K} .
$$

Proof. Given a sequence of compact subsets $K_{n} \uparrow \mathbb{R}^{d}$, we have

$$
W^{*}=\bigcup_{n \geq 0} W_{K_{n}}^{*}
$$

and the uniqueness of $v$ is immediate. For the existence of $v$, it suffices to check that for $K \subseteq K^{\prime}$ compact subsets of $\mathbb{R}^{d}$

$$
\pi^{*} \circ Q_{K}=\pi^{*} \circ\left(1_{W_{K}} Q_{K^{\prime}}\right) .
$$

Indeed, one then defines $v$ so that $1_{W_{K_{n}}^{*}} v=\pi^{*} \circ Q_{K_{n}}$ for some $K_{n} \uparrow \mathbb{R}^{d}$, noting that the restriction to $W_{K_{n}}^{*}$ of $\pi^{*} \circ Q_{K_{n+1}}$ equals $\pi^{*} \circ\left(1_{W_{K_{n}}} Q_{K_{n+1}}\right)$ which equals $\pi^{*} \circ Q_{K_{n}}$ by (2.8). Using (2.8) once again, one sees that $v$ does not depend on the sequence $K_{n}$, and (2.7) immediately follows (choosing $K_{0}=K$ ). To prove (2.8), we note that when the closed ball $B$ contains $K^{\prime}$, then by (2.5) we have

$$
\begin{aligned}
& Q_{K}=\theta_{H_{K}} \circ\left(1_{W_{K}} Q_{B}\right) \text { and } \\
& 1_{W_{K}} Q_{K^{\prime}}=1_{W_{K}} \theta_{H_{K^{\prime}}} \circ\left(1_{W_{K^{\prime}}} Q_{B}\right)=\theta_{H_{K^{\prime}}} \circ\left(1_{W_{K}} Q_{B}\right) .
\end{aligned}
$$

Hence, the images under $\pi^{*}$ of $Q_{K}$ and $1_{W_{K}^{\prime}} Q_{K^{\prime}}$ coincide, that is (2.7) holds. 
We are now reduced to the

Proof of Lemma 2.1. It suffices to show that for any continuous compactly supported function $f: \mathbb{R} \rightarrow \mathbb{R}^{d}$,

$$
\begin{gathered}
\int e_{B^{\prime}}\left(d y^{\prime}\right) E_{y^{\prime}}\left[H_{B}<\infty, e^{i \int_{0}^{H_{B}} f\left(v-H_{B}\right) \cdot X_{v} d v} E_{X_{H_{B}}}\left[e^{i \int_{0}^{\infty} f(v) \cdot X_{v} d v}\right] F^{\prime}\left(y^{\prime}, H_{B}\right)\right] \\
=\int e_{B}(d y) E_{y}^{B}\left[e^{i \int_{0}^{\infty} f(-v) \cdot X_{v} d v}\right] E_{y}\left[e^{i \int_{0}^{\infty} f(v) \cdot X_{v} d v}\right], \text { where } \\
F^{\prime}\left(y^{\prime}, t\right)=E_{y^{\prime}}^{B^{\prime}}\left[e^{i \int_{0}^{\infty} f(-v-t) \cdot X_{v} d v}\right], \text { for } y^{\prime} \in \partial B^{\prime}, t \geq 0 .
\end{gathered}
$$

Indeed the second line of (2.9) coincides with $E^{Q_{B}}\left[e^{i \int_{\mathbb{R}} f(v) \cdot X_{v} d v}\right]$, whereas by the strong Markov property at time $H_{B}$, the first line of (2.9) equals

$$
\begin{aligned}
& E^{Q_{B^{\prime}}}\left[H_{B}<\infty, e^{i \int_{\mathbb{R}} f\left(v-H_{B}\right) \cdot X_{v} d v}\right] \\
= & E^{\theta_{H_{B}} \circ\left(1\left\{H_{B}<\infty\right\} Q_{B^{\prime}}\right)}\left[e^{i \int_{\mathbb{R}} f(v) \cdot X_{v} d v}\right],
\end{aligned}
$$

and the claim (2.4) follows. We will thus prove (2.9). We denote by $P_{y, y^{\prime}}^{t}$ the Brownian bridge measure in time $t>0$, from $y$ to $y^{\prime}$ in $\mathbb{R}^{d}$, see [36], p. 137-140, and write $E_{y, y^{\prime}}^{t}$ for the corresponding expectation. We consider $y \in B^{\prime}$ and use the last exit decomposition at the last visit of $B^{\prime}$, see Theorem 2.12 of [14] and (3.39) on p. 60 of [36], to assert that under $P_{y}$, conditionally on $L_{B^{\prime}}=t$ (with $t>0)$, and $X_{L_{B^{\prime}}}=y^{\prime}$ (with $y^{\prime} \in \partial B$ ), the law of $\left(X_{L_{B^{\prime}}+v}\right)_{v \geq 0}$ and $\left(X_{v}\right)_{0 \leq v \leq t}$ are independent, respectively distributed as $P_{y^{\prime}}^{B}$ and $P_{y, y^{\prime}}^{t}$ and $\left(L_{B^{\prime}}, X_{L_{B^{\prime}}}\right)$ has distribution $p_{t}\left(y, y^{\prime}\right) e_{B^{\prime}}\left(d y^{\prime}\right) 1\{t>0\} d t$. Writing $U=B^{c}$ we find as a result that for $y$ in $B^{\prime}$

$$
\begin{aligned}
& E_{y}\left[e^{i \int_{0}^{\infty} f(-v) \cdot X_{v} d v}, H_{B}=\infty\right] \\
= & \int_{0}^{\infty} d t \int e_{B^{\prime}}\left(d y^{\prime}\right) E_{y^{\prime}}^{B^{\prime}}\left[e^{i \int_{0}^{\infty} f(-v-t) \cdot X_{v} d v}\right] \\
& \times E_{y, y^{\prime}}^{t}\left[e^{i \int_{0}^{t} f(-v) \cdot X_{v} d v}, T_{U}>t\right] p_{t}\left(y, y^{\prime}\right) \\
= & \int_{0}^{\infty} d t \int e_{B^{\prime}}\left(d y^{\prime}\right) F^{\prime}\left(y^{\prime}, t\right) E_{y^{\prime}, y}^{t}\left[e^{\int_{0}^{t} f(v-t) \cdot X_{v} d v}, T_{U}>t\right] p_{t}\left(y^{\prime}, y\right),
\end{aligned}
$$

where, in the last step, we used that the law of $\left(X_{t-} .\right)_{0 \leq-\leq t}$ under $P_{y, y^{\prime}}^{t}$ equals $P_{y^{\prime}, y}^{t}$, cf. (A.12), p. 139 of [36], that $p_{t}(\cdot, \cdot)$ is symmetric, and the notation from below (2.9). 
We introduce the notation $F(z)=\psi(z) E_{z}\left[e^{i \int_{0}^{\infty} f(v) \cdot X_{v} d v}\right]$ for $z \in \mathbb{R}^{d}$, where $\psi$ is a continuous compactly supported $[0,1]$-valued function, which equals to 1 on a neighborhood of $B$. We pick $\varepsilon>0$ and introduce the finite measure

$$
\begin{aligned}
m_{\varepsilon}(d y) & =\frac{1}{\varepsilon} \int d z F(z) P_{z}\left[H_{B}<\varepsilon, X_{\varepsilon} \in d y\right] \\
& =\frac{1}{\varepsilon} \int d z F(z) P_{z, y}^{\varepsilon}\left[H_{B}<\varepsilon\right] p_{\varepsilon}(z, y) d y .
\end{aligned}
$$

We integrate (2.10) with respect to $m_{\varepsilon}(d y)$. The first line of (2.10) yields

$$
\begin{aligned}
& \int d z F(z) \frac{1}{\varepsilon} E_{z}\left[H_{B}<\varepsilon, E_{X_{\varepsilon}}\left[H_{B}=\infty, e^{i \int_{0}^{\infty} f(-v) \cdot X_{v} d v}\right]\right] \stackrel{\text { definition } P_{\cdot}^{B}}{=} \\
& \int d z F(z) \frac{1}{\varepsilon} E_{z}\left[H_{B}<\varepsilon, P_{X_{\varepsilon}}\left[H_{B}=\infty\right] E_{X_{\varepsilon}}^{B}\left[e^{i \int_{0}^{\infty} f(-v) \cdot X_{v} d v}\right]\right] \stackrel{\text { simple Markov }}{=} \begin{array}{c}
= \\
\text { property }
\end{array} \\
& \int d z F(z) \frac{1}{\varepsilon} E_{z}\left[H_{B}<\varepsilon, H_{B} \circ \theta_{\varepsilon}=\infty, E_{X_{\varepsilon}}^{B}\left[e^{i \int_{0}^{\infty} f(-v) \cdot X_{v} d v}\right]\right]= \\
& \int d z F(z) \frac{1}{\varepsilon} E_{z}\left[0<L_{B}<\varepsilon, E_{X_{\varepsilon}}^{B}\left[e^{i \int_{0}^{\infty} f(-v) \cdot X_{v} d v}\right]\right] .
\end{aligned}
$$

By the last exit decomposition of Brownian motion starting at $z$ at the last visit of $B$, the above expression equals

$$
\begin{aligned}
& \int d z F(z) \frac{1}{\varepsilon} \int_{0}^{\varepsilon} d s p_{s}(z, y) e_{B}(d y) E_{y}^{B}\left[E_{X_{\varepsilon-s}}^{B}\left[e^{i \int_{0}^{\infty} f(-v) \cdot X_{v} d v}\right]\right] \\
= & \int e_{B}(d y) \frac{1}{\varepsilon} \int_{0}^{\varepsilon} d s\left(\int p_{s}(y, z) F(z) d z\right) E_{y}^{B}\left[E_{X_{\varepsilon-s}}^{B}\left[e^{i \int_{0}^{\infty} f(-v) \cdot X_{v} d v}\right]\right]
\end{aligned}
$$

(by symmetry of $p_{s}(\cdot, \cdot)$ ).

Using the fact that for $y \in \partial B, P_{y}^{B}$-a.s., as $u \rightarrow 0, P_{X_{u}}^{B}$ converges weakly to $P_{y}^{B}$ (one could actually also invoke the Markov property under $P_{y}^{B}$ at this point), and dominated convergence, as $\varepsilon \rightarrow 0$, the above quantity tends to

$$
\int e_{B}(d y) E_{y}\left[e^{i \int_{0}^{\infty} f(v) \cdot X_{v} d v}\right] E_{y}^{B}\left[e^{i \int_{0}^{\infty} f(-v) \cdot X_{v} d v}\right] .
$$

Using the symmetry of $p_{\varepsilon}(\cdot, \cdot)$ and the fact that for $y \in U=B^{c}, P_{z, y}^{\varepsilon}\left[H_{B}<\right.$ $\varepsilon]=P_{y, z}^{\varepsilon}\left[H_{B}<\varepsilon\right]$ (when $z \in B$, note that $P_{z}$-a.s., $\widetilde{H}_{B}=0$, and both terms 
equal 1), the last line of (2.10) after integration with respect to $m_{\varepsilon}(d y)$ yields

$$
\begin{aligned}
& \int e_{B^{\prime}}\left(d y^{\prime}\right) \int_{0}^{\infty} \frac{d t}{\varepsilon} F^{\prime}\left(y^{\prime}, t\right) \int d z d y F(z) \\
& \times E_{y^{\prime}, y}^{t}\left[e^{i \int_{0}^{t} f(v-t) \cdot X_{v} d v}, T_{U}>t\right] p_{t}\left(y^{\prime}, y\right) P_{y, z}^{\varepsilon}\left[H_{B}<\varepsilon\right] p_{\varepsilon}(y, z) \\
= & \int e_{B^{\prime}}\left(d y^{\prime}\right) \int_{0}^{\infty} \frac{d t}{\varepsilon} F^{\prime}\left(y^{\prime}, t\right) E_{y^{\prime}}\left[e^{i \int_{0}^{t} f(v-t) \cdot X_{v} d v}, t<H_{B}<t+\varepsilon, F\left(X_{t+\varepsilon}\right)\right] \\
= & \int e_{B^{\prime}}\left(d y^{\prime}\right) E_{y^{\prime}}\left[H_{B}<\infty, \frac{1}{\varepsilon} \int_{\left(H_{B}-\varepsilon\right)_{+}}^{H_{B}} e^{i \int_{0}^{t} f(v-t) \cdot X_{v} d v} F\left(X_{t+\varepsilon}\right) F^{\prime}\left(y^{\prime}, t\right) d t\right] .
\end{aligned}
$$

Applying dominated convergence as $\varepsilon \rightarrow 0$, the above quantity tends to

$$
\begin{aligned}
\int e_{B^{\prime}}\left(d y^{\prime}\right) E_{y^{\prime}}[ & H_{B}<\infty, e^{i \int_{0}^{H_{B}} f\left(v-H_{B}\right) \cdot X_{v} d v} \\
& \left.\times E_{X_{H_{B}}}\left[e^{i \int_{0}^{\infty} f(v) \cdot X_{v} d v}\right] F^{\prime}\left(y^{\prime}, H_{B}\right)\right] .
\end{aligned}
$$

Comparing (2.12) and (2.13), we have shown (2.9). This proves Lemma 2.1.

Remark 2.3. 1) When $B$ is a closed ball, for $w \in W_{B}$ we can define

$$
L_{B}(w)=\sup \left\{t \in \mathbb{R}, L_{B}(w) \in B\right\} .
$$

Applying the last exit decomposition formula, as in (2.10), to the forward term in (2.3), we see that for $A, A^{\prime} \in W_{+}$we have

$$
\begin{aligned}
& Q_{B}\left[\left(X_{-t}\right)_{t \geq 0} \in A^{\prime},\left(X_{s}\right)_{0 \leq s \leq L_{B}} \in \cdot,\left(X_{L_{B}+s}\right)_{s \geq 0} \in A\right] \\
= & \int_{0}^{\infty} d t \int e_{B}\left(d y^{\prime}\right) e_{B}(d y) P_{y^{\prime}}^{B}\left[A^{\prime}\right] P_{y^{\prime}, y}^{t}[\cdot] P_{y}^{B}[A] p_{t}\left(y^{\prime}, y\right)
\end{aligned}
$$

(where $\left(X_{s}\right)_{0 \leq s \leq L_{B}}$ is viewed as a random element of the space $\mathcal{T}$ of continuous $\mathbb{R}^{d}$-valued trajectories of positive finite duration, measurably identified with $C\left([0,1], \mathbb{R}^{d}\right) \times(0, \infty)$ via the map $\left.(w, T) \rightarrow w(\dot{\bar{T}}) \in \mathcal{T}\right)$.

From this identity using the symmetry of $p_{t}(\cdot, \cdot)$ and the already used fact that $P_{y^{\prime}, y}^{t}$ is the image of $P_{y, y^{\prime}}^{t}$ under time reversal, we find (analogously to (1.40) of [38]) that

$$
\left(X_{L_{B}-t}\right)_{t \in \mathbb{R}} \text { under } Q_{B} \text { has law } Q_{B} .
$$

One can introduce on $W^{*}$ the time inversion $w^{*} \rightarrow \stackrel{\vee}{w}^{*}$, where $\stackrel{\vee}{w}^{*}=\pi^{*}(\stackrel{v}{w})$, with $\stackrel{\vee}{w}(t)=w(-t)$ for $t \in \mathbb{R}$, and $w \in W$ arbitrary such that $\pi^{*}(w)=w^{*}$. It 
follows from (2.15) that the image $\left(\pi^{*} \circ Q_{B}\right)^{\vee}$ of $\left(\pi^{*} \circ Q_{B}\right)$ under time inversion coincides with $\pi^{*} \circ Q_{B}$. By Theorem 2.2 it follows that the image $v$ of $v$ under time inversion satisfies

$$
\stackrel{v}{v}=v \text {. }
$$

2) In an easier fashion one sees that when one considers $y \in \mathbb{R}^{d}$ and denotes by $\tau_{y}$ the translation by $-y$ on $W^{*}$, which to $w^{*}$ associates $w^{*}-y$ (defined in an obvious way), or a linear isometry $R$ of $\mathbb{R}^{d}$, and its natural operation $w^{*} \rightarrow R w^{*}$ on $W^{*}$, we find that

$$
\begin{aligned}
& v \text { is invariant under } \tau_{y}, \\
& v \text { is invariant under } R,
\end{aligned}
$$

3) When $\lambda>0$, one can define the scaling $s_{\lambda}(w)=\lambda w\left(\dot{\lambda^{2}}\right)$ for $w \in W$, and on $W^{*}$ via $s_{\lambda}\left(w^{*}\right)=\pi^{*}\left(s_{\lambda}(w)\right)$, for $w \in W$ arbitrary such that $\pi^{*}(w)=w^{*}$. From the identity valid for arbitrary $\lambda>0$ and closed ball $B$

$$
s_{\lambda} \circ Q_{B}=\lambda^{2-d} Q_{\lambda B},
$$

one finds by Theorem 2.2 that

$$
s_{\lambda} \circ v=\lambda^{2-d} v, \text { for } \lambda>0 .
$$

4) One knows that when the compact set $K\left(\subseteq \mathbb{R}^{d}\right)$ lies in the interior of the closed ball $B$, then $e_{K}(\cdot)=P_{e_{B}}\left[H_{K}<\infty, X_{H_{K}} \in \cdot\right]$, see Theorem 1.10, p. 58 of [27]. By (2.3) and (2.5) we thus see that

$$
Q_{K}\left[\left(X_{t}\right)_{t \geq 0} \in \cdot\right]=P_{e_{K}} .
$$

We can now introduce the measurable map from $W_{K}^{*}$ into $W_{+}$defined by $w^{*} \in$ $W_{K}^{*} \rightarrow w^{*, K,+}=\left(w\left(H_{K}+t\right)\right)_{t \geq 0}$, for any $w \in W_{K}$ with $\pi^{*}(w)=w^{*}$. Then, by (2.7) and (2.21) we see that

$$
\text { the image of } 1_{W_{K}^{*}} v \text { under } w^{*} \rightarrow w^{*, K,+} \text { equals } P_{e_{K}} \text {. }
$$

As a next step we introduce the canonical space for the Brownian interlacement point process, namely the space of point measures on $W^{*} \times \mathbb{R}_{+}$,

$$
\begin{aligned}
& \Omega=\left\{\omega=\sum_{i \geq 0} \delta_{\left(w_{i}^{*}, \alpha_{i}\right)}, \text { with }\left(w_{i}^{*}, \alpha_{i}\right) \in W^{*} \times[0, \infty)\right. \text { and } \\
&\left.\omega\left(W_{K}^{*} \times[0, \alpha]\right)<\infty, \text { for any compact subset } K \text { of } \mathbb{R}^{d} \text { and } \alpha \geq 0\right\} .
\end{aligned}
$$


We endow $\Omega$ with the $\sigma$-algebra $\mathcal{A}$ generated by the evaluation maps $w \rightarrow$ $\omega(B)$, for $B \in \mathcal{W}^{*} \otimes \mathcal{B}\left(\mathbb{R}_{+}\right)$, and denote by $\mathbb{P}$ the law on $(\Omega, \mathcal{A})$ of the Poisson point measure with intensity measure $v \otimes d \alpha$ on $W^{*} \times \mathbb{R}_{+}$. For $K$ compact subset of $\mathbb{R}^{d}$ and $\alpha \geq 0$, we consider the measurable function on $\Omega$ with value in the set of finite point measures on $W_{+}$

$$
\begin{gathered}
\mu_{K, \alpha}(\omega)=\sum_{i \geq 0} 1\left\{\alpha_{i} \leq \alpha, w_{i}^{*} \in W_{K}^{*}\right\} \delta_{w_{i}^{*, K,+}}, \\
\text { when } \omega=\sum_{i \geq 0} \delta_{\left(w_{i}^{*}, \alpha_{i}\right)} \in \Omega,
\end{gathered}
$$

with the notation from above (2.22). We readily see by (2.22) that

$$
\mu_{K, \alpha} \text { is a Poisson point process on } W_{+} \text {with intensity measure } \alpha P_{e_{K}} \text {. }
$$

As a direct consequence of (2.16)-(2.20) we obtain the following invariance properties.

Proposition 2.4. $\mathbb{P}$ is invariant under the following transformations on $\Omega$ :

$$
\begin{aligned}
& \omega=\sum_{i \geq 0} \delta_{\left(w_{i}^{*}, \alpha_{i}\right)} \longrightarrow \sum_{i \geq 0} \delta_{\left(w_{i}^{*}, \alpha_{i}\right)}, \\
& \omega=\sum_{i \geq 0} \delta_{\left(w_{i}^{*}, \alpha_{i}\right)} \longrightarrow \sum_{i \geq 0} \delta_{\left(w_{i}^{*}-y, \alpha_{i}\right)} \quad\left(\text { with } y \in \mathbb{R}^{d}\right), \\
& \left.\omega=\sum_{i \geq 0} \delta_{\left(w_{i}^{*}, \alpha_{i}\right)} \longrightarrow \sum_{i \geq 0} \delta_{\left(R w_{i}^{*}, \alpha_{i}\right)} \quad \text { (with R linear isometry of } \mathbb{R}^{d}\right), \\
& \left.\omega=\sum_{i \geq 0} \delta_{\left(w_{i}^{*}, \alpha_{i}\right)} \longrightarrow \sum_{i \geq 0} \delta_{\left(s_{\lambda}\left(w_{i}^{*}\right), \lambda^{2-d} \alpha_{i}\right)} \text { (with } \lambda>0\right) .
\end{aligned}
$$

When $\omega \in \Omega, \alpha \geq 0, r \geq 0$, the formula (see the beginning of Section 1 for notation)

$$
\mathcal{I}_{r}^{\alpha}(\omega)=\bigcup_{i \geq 0: \alpha_{i} \leq \alpha} \bigcup_{s \in \mathbb{R}} B\left(w_{i}(s), r\right)
$$

$$
\text { where } \omega \in \sum_{i \geq 0} \delta_{\left(w_{i}^{*}, \alpha_{i}\right)} \text { and } \pi^{*}\left(w_{i}\right)=w_{i}^{*} \text { for } i \geq 0,
$$

defines the Brownian interlacement at level $\alpha$ with radius $r$. By construction, see (2.23), this is a closed subset of $\mathbb{R}^{d}$. When $r=0$, we refer to $\mathcal{I}_{0}^{\alpha}(\omega)$ as the Brownian fabric at level $\alpha$. The terminology is truly pertinent when $d=3$, see 
(2.36) below. The complement of $\mathcal{I}_{r}^{\alpha}(\omega)$ is an open subset of $\mathbb{R}^{d}$, the vacant set at level $\alpha$ and radius $r$ :

$$
\mathcal{V}_{r}^{\alpha}(\omega)=\mathbb{R}^{d} \backslash \mathcal{I}_{r}^{\alpha}(\omega), \text { for } \omega \in \Omega, \alpha \geq 0, r \geq 0 .
$$

The set $\Sigma$ of closed (possibly empty) subsets of $\mathbb{R}^{d}$ can be endowed with the $\sigma$-algebra $\mathcal{F}$ generated by the sets $\{F \in \Sigma ; F \cap K=\emptyset\}$, where $K$ varies over the compact subsets of $\mathbb{R}^{d}$, see [25], p. 27 (incidentally, this is the Borel $\sigma$ algebra for a metrizeable topology for which $\Sigma$ is compact, see [25], p. 3 and 27). The $\Sigma$-valued map $\mathcal{I}_{r}^{\alpha}$ on $\Omega$ is measurable, and one can thus consider its law $Q_{r}^{\alpha}$ on $(\Sigma, \mathcal{F})$.

Proposition 2.5. ( $\alpha \geq 0, r \geq 0, y \in \mathbb{R}^{d}, R$ linear isometry, $\lambda>0$ ) $Q_{r}^{\alpha}$ is determined by the identity

$$
\begin{gathered}
Q_{r}^{\alpha}(\{F \in \Sigma ; F \cap K=\emptyset\})=\mathbb{P}\left[\mathcal{I}_{r}^{\alpha} \cap K=\emptyset\right]=e^{-\alpha \operatorname{cap}\left(K_{r}\right)}, \\
\text { for } K \subseteq \mathbb{R}^{d} \text { compact, }
\end{gathered}
$$

where $K_{r}=K+B(0, r)$ the set of points within $|\cdot|$-distance at most $r$ from $K$. Moreover, under $\mathbb{P}$,

$$
\begin{array}{ll}
\mathcal{I}_{r}^{\alpha}+\text { y has same law as } \mathcal{I}_{r}^{\alpha} & \text { (translation invariance), } \\
R\left(\mathcal{I}_{r}^{\alpha}\right) \text { has same law as } \mathcal{I}_{r}^{\alpha} & \text { (isotropy), } \\
\lambda \mathcal{I}_{r}^{\alpha} \text { has same law as } \mathcal{I}_{\lambda r}^{\lambda^{2-d} \alpha} & \text { (scaling), } \\
\mathcal{I}_{0}^{\alpha} \text { is a.s.-connected, when } d=3, & \\
\text { and disconnected, when } d \geq 4 \text { and } \alpha>0 .
\end{array}
$$

Proof. Note that $\left\{\mathcal{I}_{r}^{\alpha} \cap K=\emptyset\right\}=\left\{\omega \in \Omega ; \omega\left(W_{K_{r}}^{*} \times[0, \alpha]\right)=0\right\}$, so that

$$
\mathbb{P}\left[\mathcal{I}_{r}^{\alpha} \cap K=\emptyset\right]=c^{-\alpha \nu\left(W_{K_{r}}^{*}\right) \stackrel{(2.7),(2.21)}{=}} e^{-\alpha \operatorname{cap}\left(K_{r}\right)} \text {, whence (2.32). }
$$

The identity (2.32) determines $Q_{r}^{\alpha}$ on a $\pi$-system generating $\mathcal{F}$, and the first claim follows. The identities (2.33)-(2.35) are direct consequences of (2.27)(2.29) (alternatively, they can be derived from (2.32)). The last claim is a direct consequence of the fact that for any closed ball $B, Q_{B}(d w) \otimes Q_{B}\left(d w^{\prime}\right)$-a.s., $w(\mathbb{R}) \cap w^{\prime}(\mathbb{R}) \neq \emptyset$, when $d=3$, but $w(\mathbb{R}) \cap w^{\prime}(\mathbb{R})=\emptyset$, when $d \geq 4$, as a consequence of the fact that Brownian paths meet each other when $d=3$, and, when starting from different points, miss each other when $d \geq 4$, see [11] (when $d \geq 4$ we also use (2.15) to take care of the bilateral nature of the paths). 
As a last topic of this section, we introduce the occupation-time measure of Brownian interlacements at level $\alpha \geq 0$ and discuss some of its properties. It is the locally finite (or Radon) measure on $\mathbb{R}^{d}$ defined for $\omega \in \Omega, \alpha \geq 0$, $A \in \mathcal{B}\left(\mathbb{R}^{d}\right)$ by

$$
\begin{aligned}
\mathcal{L}_{\alpha}(\omega)(A)= & \sum_{i \geq 0: \alpha_{i} \leq \alpha} \int_{\mathbb{R}} 1\left\{w_{i}(s) \in A\right\} d s, \text { where } \omega=\sum_{i \geq 0} \delta_{\left(w_{i}^{*}, \alpha_{i}\right)} \\
& \text { with } \pi^{*}\left(w_{i}\right)=w_{i}^{*} \text {, for } i \geq 0, \\
= & \left\langle\omega, f_{A} \otimes 1_{[0, \alpha]}\right\rangle,
\end{aligned}
$$

where $f_{A}\left(w^{*}\right)=\int_{\mathbb{R}} 1_{A}(w(s)) d s$, for $w \in W$ arbitrary such that $\pi^{*}(w)=w^{*}$, and $\langle\omega, h\rangle$ stands for the integral of $h$ with respect to $\omega$. Note that by the choice of $\Omega$ and $W^{*}$, the expression in (2.37) is finite when $A$ is a bounded set. From the second line of (2.37), the dependence in $\omega$ is $\mathcal{A}$-measurable, and $\mathcal{L}_{\alpha}$ defines a random measure on $\mathbb{R}^{d}$, see Chapter 1 of [19]. Further when $A \in B\left(\mathbb{R}^{d}\right)$ is bounded and $B$ a closed ball containing $A$, then for $\alpha \geq 0$

$$
\begin{aligned}
\mathbb{E}\left[\mathcal{L}_{\alpha}(\omega)(A)\right] & =\mathbb{E}\left[\left\langle\omega, f_{A} \otimes 1_{[0, \alpha]}\right]\right] \\
& =\alpha\left\langle\nu, f_{A}\right\rangle \stackrel{(2.7),(2.3)}{=} \alpha E_{e_{B}}\left[\int_{0}^{\infty} 1_{A}\left(X_{s}\right) d s\right] \\
& =\alpha \int e_{B}(d y) G\left(y, y^{\prime}\right) 1_{A}\left(y^{\prime}\right) d y^{\prime} \\
& =\alpha|A|(\text { with }|A| \text { the Lebesgue measure of } A),
\end{aligned}
$$

since $\int e_{B}(d y) G\left(y, y^{\prime}\right)=1$ for $y^{\prime} \in B$, by (2.2), (2.1). In other words:

the intensity measure of $\mathcal{L}_{\alpha}$ equals $\alpha d y$.

Observe also that by inspection of (2.37)

$$
\text { the support of } \mathcal{L}_{\alpha} \text { coincides with } \mathcal{I}_{0}^{\alpha} \text {. }
$$

The next result will be very useful and provides an expression for the Laplace transform of $\mathcal{L}_{\alpha}$.

Proposition 2.6. When $V$ is a bounded, measurable, compactly supported function on $\mathbb{R}^{d}$, and

$$
\|G|V|\|_{L^{\infty}\left(\mathbb{R}^{d}\right)}<1 \text { (see (1.10) for notation), }
$$

then $I-G V$ is a bounded invertible operator on $L^{\infty}\left(\mathbb{R}^{d}\right)$ and for any $\alpha \geq 0$,

$$
\mathbb{E}\left[\exp \left\{\left\langle\mathcal{L}_{\alpha}, V\right\rangle\right\}\right]=\exp \left\{\alpha\left\langle V,(I-G V)^{-1} 1\right\rangle\right\},
$$

with the notation (1.9) and $\left\langle\mathcal{L}_{\alpha}, V\right\rangle$ the integral of $V$ with respect to $\mathcal{L}_{\alpha}$. 
Proof. Up to routine modifications, this follows by similar arguments as Theorem 2.1 of [39].

Remark 2.7. As a direct application of the invariance property (2.29) and the definition (2.9) we see that for $\alpha \geq 0$, the occupation-time measure $\mathcal{L}_{\alpha}$ has the following scaling invariance

$$
\mathcal{L}_{\alpha} \stackrel{\text { law }}{=} \lambda^{2} h_{\lambda} \circ \mathcal{L}_{\lambda^{d-2} \alpha}, \text { for } \lambda>0,
$$

where $h_{\lambda}(y)=\lambda y$ denotes the homothety of ratio $\lambda$ on $\mathbb{R}^{d}$. One can also recover the identity (2.43) from (2.42).

\section{Scaling limits of occupation times}

In this section we study the scaling limit of the random field of occupation times of random interlacements on $\mathbb{Z}^{d}, d \geq 3$. In the constant intensity regime (0.2), we show that $\mathcal{L}^{N}$ (see (0.1)) converges in distribution to the occupation-time measure of Brownian interlacements, see Theorem 3.2. In the high intensity regime (0.4), we show that $\widehat{\mathcal{L}}^{N}$ (see (0.5)) converges in distribution to the massless Gaussian free field, see Theorem 3.3 and Corollary 3.5.

We tacitly endow the set of Radon measures on $\mathbb{R}^{d}$ with the topology of the vague convergence (see A.7 of [19]). Given a positive sequence $\left(u_{N}\right)_{N \geq 1}$, it follows from (1.12) that

$$
\overline{\mathbb{E}}\left[\mathcal{L}^{N}\left([0,1)^{d}\right)\right]=\frac{1}{d} N^{d-2} u_{N} .
$$

This quantity equals $\alpha(>0)$ in the constant intensity regime (0.2), and tends to $\infty$ in the high intensity regime (0.4). The low intensity regime (when $N^{d-2} u_{N} \rightarrow$ 0 ) leads to a convergence in distribution of $\mathcal{L}^{N}$ to the null measure on $\mathbb{R}^{d}$, and we will only focus on the constant and high intensity regimes.

We begin with some preparation. We consider an integer $M \geq 1$, and a continuous function $V$ on $\mathbb{R}^{d}$ with support in $C_{M}=[-M, M]^{d}$.

We recall the notation (1.8), (1.10) from Section 1. With a slight abuse of notation, we still denote by $V$ the restriction of $V$ to $\mathbb{L}_{N}$ (see (1.4)).

Proposition 3.1. (under (3.2))

$$
\begin{aligned}
& \sup _{N \geq 1}\left\|G_{N}|V|\right\|_{L^{\infty}\left(\mathbb{L}_{N}\right)} \leq c_{0}(M)\|V\|_{L^{\infty}\left(C_{M} \cap \mathbb{L}_{N}\right)} \leq c_{0}(M)\|V\|_{L^{\infty}\left(\mathbb{R}^{d}\right)} . \\
& \lim _{N}\left\langle V,\left(G_{N} V\right)^{n-1} 1\right\rangle_{\mathbb{L}_{N}}=\left\langle V,(G V)^{n-1} 1\right\rangle, \text { for all } n \geq 1 .
\end{aligned}
$$


Proof. We begin with the proof of (3.3), and assume, without loss of generality, that $\|V\|_{L^{\infty}\left(C_{M} \cap \mathbb{L}_{N}\right)}=1$. By a classical harmonicity (or martingale) argument one sees that

$$
\sup _{y \in \mathbb{L}_{N}}\left|G_{N}\right| V|(y)|=\sup _{y \in C_{M} \cap \mathbb{L}_{N}}\left|G_{N}\right| V|(y)|,
$$

since $V$ is supported in $C_{M}$. It then follows from (1.2), (1.8) that

$$
\begin{aligned}
\sup _{y \in C_{M} \cap \mathbb{L}_{N}}\left|G_{N}\right| V|(y)| & \leq \frac{c}{N^{2}}\left(1+\sum_{0<|x|_{\infty} \leq 2 M N} \frac{1}{|x|^{d-2}}\right) \\
& \left.\leq c^{\prime} M^{2} \text { ( } x \text { belongs to } \mathbb{Z}^{d} \text { in the sum }\right),
\end{aligned}
$$

and the claim (3.3) follows. Note that the continuity of $V$ was not needed for the proof of (3.3). We then turn to the proof of (3.4). As we now explain, it suffices to show that for all bounded continuous functions $W$ on $\mathbb{R}^{d}$,

$$
\lim _{N} \sup _{y \in C_{M} \cap \mathbb{L}_{N}}\left|\left(G_{N} V W\right)(y)-(G V W)(y)\right|=0 .
$$

Indeed, once (3.6) is established, we note that $G V W$ is bounded continuous on $\mathbb{R}^{d}$ as a convolution of the locally integrable function $G(\cdot)$ (see (1.3)) with the compactly supported continuous function $V W$. Then, we observe that for $n \geq 1$,

$$
\begin{aligned}
& \sup _{y \in C_{M} \cap \mathbb{L}_{N}}\left|\left(G_{N} V\right)^{n} 1(y)-(G V)^{n} 1(y)\right| \\
\leq & \sup _{y \in C_{M} \cap \mathbb{L}_{N}}\left|\left(G_{N} V\right)\left(\left(G_{N} V\right)^{n-1} 1-(G V)^{n-1} 1\right)(y)\right| \\
+ & \sup _{y \in C_{M} \cap \mathbb{L}_{N}}\left|\left(G_{N} V(G V)^{n-1} 1\right)(y)-\left(G V(G V)^{n-1} 1\right)(y)\right| .
\end{aligned}
$$

Using induction over $n \geq 1$, we see that the last term tends to zero with $N$, by (3.6) and the observation below (3.6), and the first term after the inequality sign tends to zero with $N$ by the induction hypothesis and the first inequality in (3.3). Thus, once (3.6) is proved, it follows that

$$
\sup _{C_{M} \cap \mathbb{L}_{N}}\left|\left(G_{N} V\right)^{n-1} 1(\cdot)-(G V)^{n-1} 1(\cdot)\right| \underset{N}{\longrightarrow} 0, \text { for each } n \geq 1 .
$$

The claim (3.4) now follows by a straightforward Riemann sum approximation.

We now prove (3.6) and introduce the shorthand $F=V W$. The function $F$ is continuous on $\mathbb{R}^{d}$ with support in $C_{M}$. Then, for $\gamma \in(0,1), N \geq 1$, and 
$y \in C_{M} \cap \mathbb{L}_{N}$, we have

$$
\begin{aligned}
& \left|G_{N} F(y)-G F(y)\right|=\left|\frac{1}{N^{d}} \sum_{y^{\prime} \in \mathbb{L}_{N}} g_{N}\left(y^{\prime}\right) F\left(y-y^{\prime}\right)-\int_{\mathbb{R}^{d}} G(y) F\left(y-y^{\prime}\right) d y^{\prime}\right| \\
\leq & \left(\frac{1}{N^{d}} \sum_{\left|y^{\prime}\right|_{\infty} \leq \gamma} g_{N}\left(y^{\prime}\right)+\int_{\left|y^{\prime}\right|_{\infty} \leq \gamma} G\left(y^{\prime}\right) d y^{\prime}\right)\|F\|_{L^{\infty}\left(\mathbb{R}^{d}\right)} \\
& +\left|\frac{1}{N^{d}} \sum_{\gamma \leq\left|y^{\prime}\right|_{\infty} \leq 2 M} g_{N}\left(y^{\prime}\right) F\left(y-y^{\prime}\right)-\int_{\gamma \leq\left|y^{\prime}\right|_{\infty} \leq 2 M} G\left(y^{\prime}\right) F\left(y-y^{\prime}\right) d y^{\prime}\right| .
\end{aligned}
$$

The functions $G(\cdot) F(y-\cdot)$ are uniformly continuous on $\left\{y^{\prime} \in \mathbb{R}^{d} ; \gamma \leq\left|y^{\prime}\right|_{\infty} \leq\right.$ $2 M$ \} uniformly in $y \in C_{M}$. By (1.7) and a Riemann sum approximation argument we see that the last term of (3.8) tends to zero uniformly in $y \in C_{M} \cap \mathbb{L}_{N}$. On the other hand, the second line of (3.8) is bounded by

$$
\left(\frac{c}{N^{2}}\left(\gamma^{2} N^{2}+1\right)+c^{\prime} \gamma^{2}\right)\|F\|_{L^{\infty}\left(\mathbb{R}^{d}\right)} .
$$

Taking a limsup in $N$, and then letting $\gamma$ tend to zero, we obtain (3.6). The claim (3.4) now follows, and this completes the proof of Proposition 3.1.

We are now ready to state the convergence result for the law of $\mathcal{L}^{N}$ in the constant intensity regime (i.e. $N^{d-2} u_{N}=d \alpha$, see (0.2)). The definition of $\mathcal{L}_{\alpha}$ appears in (2.37).

Theorem 3.2. (under (0.2))

$$
\mathcal{L}^{N} \text { converges in distribution to } \mathcal{L}_{\alpha} \text {, as } N \rightarrow \infty \text {. }
$$

Proof. By Theorem 4.2, p. 22 of [19], it suffices to show that for any continuous compactly supported function $V$ on $\mathbb{R}^{d}$

$$
\left\langle\mathcal{L}^{N}, V\right\rangle \text { converges in distribution to }\left\langle\mathcal{L}_{\alpha}, V\right\rangle \text {, as } N \rightarrow \infty .
$$

Without loss of generality we assume that $V$ satisfies (3.2) and $\|V\|_{L^{\infty}\left(\mathbb{R}^{d}\right)} \leq 1$. By (1.11) and (3.3) we see that for $|z|<\frac{1}{c_{0}}$ (with $c_{0}$ from (3.3)) and $N \geq 1$,

$$
\begin{aligned}
\overline{\mathbb{E}}\left[\exp \left\{z\left|\mathcal{L}^{N}, V\right\rangle\right\}\right] & =\exp \left\{\alpha\left\langle z V,\left(I-z G_{N} V\right)^{-1} 1\right\rangle_{\mathbb{L}_{N}}\right\} \\
& =\exp \left\{\alpha \sum_{n \geq 1} z^{n}\left\langle V,\left(G_{N} V\right)^{n-1} 1\right\rangle_{\mathbb{L}_{N}}\right\} .
\end{aligned}
$$


In particular this shows (with (3.3)) that

$$
\sup _{N \geq 1} \overline{\mathbb{E}}\left[\cosh \left(r\left\langle\mathcal{L}^{N}, V\right\rangle\right)\right]<\infty \text {, when } r<c_{0}^{-1} .
$$

Thus, the laws of $\left\langle\mathcal{L}^{N}, V\right\rangle, N \geq 1$, are tight, and the random variables $e^{z\left\langle\mathcal{L}^{N}, V\right\rangle}$, $N \geq 1,|\operatorname{Re} z| \leq r\left(<c_{0}^{-1}\right)$, are uniformly integrable. Hence, if along some subsequence $N_{k}, k \geq 1$, the random variables $\left\langle\mathcal{L}^{N}, V\right\rangle$ converge in distribution to a random variable $U$, it follows from Theorem 5.4, p. 32 of [3], that for $|z|<c_{0}^{-1}$ in $\mathbb{C}$ one has

$$
\begin{aligned}
& E\left[e^{z U}\right]=\lim _{k} \overline{\mathbb{E}}\left[e^{z\left\langle\mathcal{L}^{N_{k}}, V\right\rangle}\right] \\
& \stackrel{(3.11)}{=} \lim _{k} \exp \left\{\alpha \sum_{n \geq 1} z^{n}\left\langle V,\left(G_{N_{k}} V\right)^{n-1} 1\right\rangle_{\mathbb{L}_{N}}\right\} \\
& \stackrel{(3.3),(3.4)}{=} \exp \left\{\alpha \sum_{n \geq 1} z^{n}\left\langle V,(G V)^{n-1} 1\right\rangle\right\} \stackrel{(2.42)}{=} \mathbb{E}\left[e^{z\left\langle\mathcal{L}_{\alpha}, V\right\rangle}\right],
\end{aligned}
$$

where in the last step we have used the fact that $\|G|V|\|_{L^{\infty}\left(\mathbb{R}^{d}\right)} \leq c_{0}$ as a result of (3.3) and (3.6). By analyticity, the first and the last expression in (3.13) are equal in the strip $\mid$ Rez $\mid<c_{0}^{-1}$ of the complex plane. Hence $U$ and $\left\langle\mathcal{L}_{\alpha}, V\right\rangle$ have the same characteristic function, and we have shown (3.10). This completes the proof of Theorem 3.2.

We now turn to the discussion of the high intensity regime (0.4) (i.e. $\left.N^{d-2} u_{N} \rightarrow \infty\right)$, and recall the definition of the random signed measure $\widehat{\mathcal{L}}^{N}$ from $(0.5)$ :

$$
\begin{aligned}
& \widehat{\mathcal{L}}^{N}=\sqrt{\frac{d}{2 N^{d-2} u_{N}}}\left(\mathcal{L}^{N}-\mathbb{E}\left[\mathcal{L}^{N}\right]\right) \\
& \stackrel{(1.12)}{=} \sqrt{\frac{d}{2 N^{d-2} u_{N}}}\left(\mathcal{L}^{N}-\frac{u_{N}}{d N^{2}} \sum_{y \in \mathbb{L}_{N}} \delta_{y}\right) .
\end{aligned}
$$

For a bounded measurable function $V$ on $\mathbb{R}^{d}$ with compact support, the notation $\left\langle\widehat{\mathcal{L}}^{N}, V\right\rangle$ will refer to the integral of $V$ with respect to $\widehat{\mathcal{L}}^{N}$. In essence, the next result states that $\widehat{\mathcal{L}}^{N}$ tends in distribution to the massless Gaussian free field, see (1.15). This fact will be made precise in Corollary 3.5. 
Theorem 3.3. (under (0.4)) When $V$ is continuous compactly supported function on $\mathbb{R}^{d}$, then, as $N \rightarrow \infty$,

$\left\langle\widehat{\mathcal{L}}^{N}, V\right\rangle$ converges in distribution to a centered Gaussian variable

$$
\text { with variance } E(V, V) \stackrel{(1.14)}{=} \int V(y) G\left(y-y^{\prime}\right) V\left(y^{\prime}\right) d y d y^{\prime} \text {. }
$$

Proof. Without loss of generality, we assume, for convenience, that (3.2) holds and that $\|V\|_{L^{\infty}\left(\mathbb{R}^{d}\right)} \leq 1$. Further, we use the shorthand $a_{N}=\left(\frac{2}{d} N^{d-2} u_{N}\right)^{\frac{1}{2}}$, and assume that $a_{N} \geq 1$, for all $N \geq 1$ (this possibly involves the replacement of finitely many $\left.u_{N}\right)$. We then see that for $|z|<c_{0}^{-1}$ and $N \geq 1$,

$$
\begin{array}{r}
\overline{\mathbb{E}}\left[e^{z\left\langle\widehat{\mathcal{L}}^{N}, V\right\rangle}\right]=\overline{\mathbb{E}}\left[\exp \left\{\frac{z}{a_{N}}\left(\left\langle\mathcal{L}^{N}, V\right\rangle-\frac{u_{N}}{d} N^{d-2}\langle V, 1\rangle_{\mathbb{L}_{N}}\right)\right\}\right] \\
\stackrel{(1.11),(3.3)}{=} \exp \left\{\frac{u_{N}}{d} N^{d-2} \sum_{n \geq 2} \frac{z^{n}}{a_{N}^{n}}\left\langle V,\left(G_{N} V\right)^{n-1} 1\right\rangle_{\mathbb{L}_{N}}\right\} .
\end{array}
$$

By Proposition 3.1 and the fact that $\frac{u_{N}}{d} N^{d-2} / a_{N}^{n}$ equals $\frac{1}{2}$ when $n=2$ and is bounded by $\frac{1}{2}$ and converges to zero for $N$ tending to infinity, when $n \geq 3$, we find that

$$
\overline{\mathbb{E}}\left[e^{z\left\langle\widehat{\mathbb{L}}^{N}, V\right\rangle}\right] \underset{N \rightarrow \infty}{\longrightarrow} e^{\frac{z^{2}}{2}\langle V, G V\rangle} \text { for }|z|<c_{0}^{-1} .
$$

The claim (3.15) then follows by similar arguments as below (3.13).

Remark 3.4. By (1.12) one sees that for any $N \geq 1$

$$
\overline{\mathbb{E}}\left[\sum_{y \in \mathbb{L}_{N}}(1+|y|)^{-(d+1)} L_{N y, u_{N}}\right]<\infty .
$$

Thus on a set of full $\overline{\mathbb{P}}$-measure, $(1+|y|)^{-(d+1)}$, and hence all functions $V \in$ $S\left(\mathbb{R}^{d}\right)$, are $\widehat{\mathcal{L}}^{N}$-integrable. Redefining $\widehat{\mathcal{L}}^{N}$ on a negligible set as (for instance) being equal to zero, we see that $\widehat{\mathcal{L}}^{N}(\omega)$ is a tempered distribution for each $\omega \in$ $\bar{\Omega}$, and $\left\langle\widehat{\mathcal{L}}^{N}, V\right\rangle$ a random variable on $(\bar{\Omega}, \overline{\mathcal{A}}, \overline{\mathbb{P}})$ for each $V \in S\left(\mathbb{R}^{d}\right)$. Hence, we can also view $\widehat{\mathcal{L}}^{N}$ as an $S^{\prime}\left(\mathbb{R}^{d}\right)$-valued random variable on $(\bar{\Omega}, \overline{\mathcal{A}}, \overline{\mathbb{P}})$.

In the next result about convergence in distribution of $\widehat{\mathcal{L}}^{N}$, the space $S^{\prime}\left(\mathbb{R}^{d}\right)$ is tacitly endowed with the strong topology (see p. 60 of [13], or p. 5, 6 of [17]). We recall the notation $P^{G}$ for the law on $S^{\prime}\left(\mathbb{R}^{d}\right)$ of the massless Gaussian free field (see (1.15)). The next result states the convergence of $\widehat{\mathcal{L}}_{N}$ in distribution to the massless Gaussian free field in the high intensity regime.

Corollary 3.5. (under (0.4))

$$
\widehat{\mathcal{L}}^{N} \text { converges in law to } P^{G} \text { as } N \rightarrow \infty \text {. }
$$


Proof. By the Lévy continuity theorem on $S^{\prime}\left(\mathbb{R}^{d}\right)$ (see Théorème 2, p. 516 of [26], and also Théorème 3.6.5, p. 69 of [12]), it suffices to show that for each $V \in S\left(\mathbb{R}^{d}\right)$, the variables $\left\langle\widehat{\mathcal{L}}^{N}, V\right\rangle$ converge in distribution to $\langle\Phi, V\rangle$ (under $P^{G}$ ), namely a centered Gaussian variable with variance $E(V, V)$, (see (1.15)).

We thus consider $V \in S\left(\mathbb{R}^{d}\right)$ and a continuous [0,1]-valued compactly supported function $\chi_{L}$ on $\mathbb{R}^{d}$ equal to 1 on $C_{2}$ (see (3.2) for notation). We write $\chi_{L}(\cdot)=\chi(\dot{\dot{L}})$ for $L \geq 1$ and define $V_{L}=\chi_{L} V$.

By dominated convergence $E\left(V_{L}, V_{L}\right) \rightarrow E(V, V)$, as $L \rightarrow \infty$. By Theorem 3.3 our claim (3.18) will thus follow once we show that

$$
\lim _{L \rightarrow \infty} \sup _{N \geq 1} \overline{\mathbb{E}}\left[\left\langle\widehat{\mathcal{L}}^{N}, V-V_{L}\right\rangle^{2}\right]=0 .
$$

By differentiating (3.16) twice in $z$ at the origin and approximating $V$ by $V_{L^{\prime}}$, with $L^{\prime} \rightarrow \infty$, we see from Fatou's Lemma that

$$
\overline{\mathbb{E}}\left[\left\langle\widehat{\mathcal{L}}^{N}, V-V_{L}\right\rangle^{2}\right] \leq\left\langle V-V_{L}, G_{N}\left(V-V_{L}\right)\right\rangle_{L_{N}} \text {, for } N \geq 1, L \geq 1 .
$$

Applying (3.3) to $\chi(\cdot)|V|(\cdot+a)$ for $a \in \mathbb{Z}^{d}$ and using that $\sup \left\{g_{N}(y) ; y \in \mathbb{L}_{N}\right.$, $\left.|y|_{\infty} \geq 1\right\}$ is uniformly bounded in $N$ (see (1.7)), we see that

$$
\rho=\sup _{N \geq 1}\left\|G_{N}|V|\right\|_{L^{\infty}\left(\mathbb{L}_{N}\right)}<\infty,
$$

and that

$$
\begin{gathered}
\left\langle V-V_{L},\left.G_{N}\left(V-V_{L}\right)\right|_{\mathbb{L}_{N}}\right. \\
\leq c \rho \sum_{a \in \mathbb{Z}^{d}} \sup _{y \in a+[0,1)^{d}}\left|\left(1-\chi_{L}(y)\right) V(y)\right| \underset{L \rightarrow \infty}{\longrightarrow} 0 .
\end{gathered}
$$

The claim (3.19) follows, and this completes the proof of Corollary 3.5.

\section{Scaling limits via the isomorphism theorem}

In this short section we revisit Theorem 3.3 under the perspective of the isomorphism theorem stated in (0.6). This offers a different route to Theorem 3.3 for sequences $\left(u_{N}\right)_{N \geq 1}$ in a "sufficiently high intensity regime". The special role of dimension 3 where we recover the full range (0.4) will be highlighted. Notation for the Gaussian free field on $\mathbb{Z}^{d}$ have been introduced at the end of Section 1. In particular, $\left(\varphi_{x}\right)_{x \in \mathbb{Z}^{d}}$ stands for the canonical field on $\mathbb{R}^{\mathbb{Z}^{d}}$ and $P^{g}$ for the canonical law of the Gaussian free field. 
We introduce the random signed measure on $\mathbb{R}^{d}$

$$
\Phi^{N}=\frac{1}{\sqrt{d} N^{\frac{d}{2}+1}} \sum_{x \in \mathbb{Z}^{d}} \varphi_{x} \delta_{\frac{x}{N}} .
$$

Given a continuous compactly supported function $V$ on $\mathbb{R}^{d}$ and a positive sequence $\left(u_{N}\right)_{N \geq 1}$ the identity (0.6) implies that under $P^{g} \otimes \bar{P}$

$$
\begin{aligned}
& \frac{1}{2} \sum_{z \in \mathbb{Z}^{d}} V\left(\frac{x}{N}\right)\left(\varphi_{x}^{2}-g(0)\right)+\sum_{x \in \mathbb{Z}^{d}} V\left(\frac{x}{N}\right)\left(L_{x, u_{N}}-u_{N}\right) \stackrel{\text { law }}{=} \\
& \frac{1}{2} \sum_{z \in \mathbb{Z}^{d}} V\left(\frac{x}{N}\right)\left(\varphi_{x}^{2}-g(0)\right)+\sqrt{2 u_{N}} \sum_{x \in \mathbb{Z}^{d}} V\left(\frac{x}{N}\right) \varphi_{x} .
\end{aligned}
$$

With the notation (0.5) and (4.1), this identity can be rewritten under the form

$$
\begin{aligned}
& \frac{1}{2} \sum_{z \in \mathbb{Z}^{d}} V\left(\frac{x}{N}\right)\left(\varphi_{x}^{2}-g(0)\right)+\sqrt{2 d u_{N}} N^{\frac{d}{2}+1}\left\langle\widehat{\mathcal{L}}^{N}, V\right\rangle \stackrel{\text { law }}{=} \\
& \frac{1}{2} \sum_{z \in \mathbb{Z}^{d}} V\left(\frac{x}{N}\right)\left(\varphi_{x}^{2}-g(0)\right)+\sqrt{2 d u_{N}} N^{\frac{d}{2}+1}\left\langle\Phi^{N}, V\right\rangle .
\end{aligned}
$$

Importantly, note that $\widehat{\mathcal{L}}^{N}$ involves $u_{N}$ in its definition, but $\Phi^{N}$ does not (cf. (4.1)). In the next lemma we look at the size of the terms in (4.3) which involve the Gaussian free field. To this end, we introduce the sequence

$$
b_{N}= \begin{cases}N^{4} & \text { when } d=3, \\ N^{4} \log N & \text { when } d=4, \\ N^{d} & \text { when } d \geq 5 .\end{cases}
$$

\section{Lemma 4.1.}

$\left\langle\Phi^{N}, V\right\rangle$ is a centered Gaussian variable with variance $\left\langle V, G_{N} V\right\rangle_{\mathbb{L}_{N}}$.

$\left\langle\Phi^{N}, V\right\rangle$ converges in distribution to a centered Gaussian variable

$$
\begin{gathered}
\text { with variance } \int V(y) G\left(y-y^{\prime}\right) V\left(y^{\prime}\right) d y d y^{\prime} \text {, as } N \rightarrow \infty . \\
\frac{1}{b_{N}} E^{P^{g}}\left[\left(\sum_{x \in \mathbb{Z}^{d}} V\left(\frac{x}{N}\right)\left(\varphi_{x}^{2}-g(0)\right)\right)^{2}\right] \text { has a positive limit when } N \rightarrow \infty .
\end{gathered}
$$


Proof. We begin with (4.5) and note that $\left\langle\Phi^{N}, V\right\rangle$ is a centered Gaussian variable with variance

$$
\frac{1}{d N^{d+2}} \sum_{x, x^{\prime} \in \mathbb{Z}^{d}} V\left(\frac{x}{N}\right) g\left(x, x^{\prime}\right) V\left(\frac{x^{\prime}}{N}\right) \stackrel{(1.5),(1.6)}{=}\left\langle V, G_{N} V\right\rangle_{\mathbb{L}_{N}},
$$

whence the claim.

Next, (4.6) is an immediate consequence of (4.5) and (3.4) (with $n=2$ ). We then turn to (4.7). We note that from Lemma 5.2.6, p. 201 of [24], one has the identity

$$
E^{P^{g}}\left[\left(\varphi_{x}^{2}-g(0)\right)\left(\varphi_{x^{\prime}}^{2}-g(0)\right)\right]=2 g^{2}\left(x, x^{\prime}\right), \text { for } x, x^{\prime} \in \mathbb{Z}^{d} .
$$

As a result we find that

$$
\begin{aligned}
& \frac{1}{b_{N}} E^{P^{g}}\left[\left(\sum_{x \in \mathbb{Z}^{d}} V\left(\frac{x}{N}\right)\left(\varphi_{x}^{2}-g(0)\right)\right)^{2}\right] \\
& =\frac{2}{b_{N}} \sum_{x, x^{\prime} \in \mathbb{Z}^{d}} V\left(\frac{x}{N}\right) g^{2}\left(x-x^{\prime}\right) V\left(\frac{x^{\prime}}{N}\right) .
\end{aligned}
$$

When $d=3, G(y)=c|y|^{-1}$ (see (1.3)) is locally square integrable on $\mathbb{R}^{3}$, and the above quantity equals (see (1.6))

$$
\frac{18}{N^{6}} \sum_{y, y^{\prime} \in \mathbb{L}_{N}} V(y) g_{N}^{2}\left(y-y^{\prime}\right) V\left(y^{\prime}\right) \underset{N \rightarrow \infty}{\longrightarrow} 18 \int V(y) G^{2}\left(y-y^{\prime}\right) V\left(y^{\prime}\right) d y d y^{\prime},
$$

as can be seen by separately considering the terms $\left|y-y^{\prime}\right| \geq \gamma$ and $\left|y-y^{\prime}\right|<\gamma$, with $y, y^{\prime} \in \mathbb{L}_{N}$, in the spirit of what was done below (3.8), letting first $N$ go to infinity, and then $\gamma$ go to zero. Note that the integral in (4.10) is equal to (with $p_{t}(\cdot, \cdot)$ the Brownian transition density, see above (2.1)):

$$
\int_{\mathbb{R}_{+} \times \mathbb{R}_{+} \times \mathbb{R}^{d} \times \mathbb{R}^{d}} d s d t d z d z^{\prime}\left(\int_{\mathbb{R}^{d}} d y V(y) p_{\frac{s}{2}}(y, z) p_{\frac{t}{2}}\left(y, z^{\prime}\right)\right)^{2}
$$

by a similar calculation as in Proposition 4.8, p. 75 of [36]. This quantity is positive when $V$ is not identically equal to zero, see the bottom of p. 75 of [36] for a similar argument.

When $d=4, b_{N}=N^{4} \log N$, and given $L \geq 1$ and $\gamma>0$, the contribution in the right-hand side of (4.9) of the terms with $\left|x-x^{\prime}\right| \leq L$ or $\left|x-x^{\prime}\right| \geq$ $\gamma N$ is $O\left(\frac{1}{\log N}\right)$, as $N$ tends to infinity, by (1.3). Combined with the fact that 
$\frac{1}{\log N} \sum_{0<|x| \leq N} \frac{1}{|x|^{4}}$ tends to a positive limit, when $N$ tends to infinity (as can be seen by looking at the difference of the sum and the integral $\left.\int_{B(0, N) \backslash B(0,1)} \frac{d x}{|x|^{4}}\right)$, we find that when $d=4$,

$$
\frac{2}{N^{4} \log N} \sum_{x, x^{\prime} \in \mathbb{Z}^{4}} V\left(\frac{x}{N}\right) g^{2}\left(x-x^{\prime}\right) V\left(\frac{x^{\prime}}{N}\right) \underset{N}{\longrightarrow} c \int_{\mathbb{R}^{4}} V^{2}(y) d y .
$$

Finally, when $d \geq 5$, the sum $\sum_{x \in \mathbb{Z}^{d}} g^{2}(x)$ converges by (1.3), and hence

$$
\frac{2}{N^{d}} \sum_{x, x^{\prime} \in \mathbb{Z}^{4}} V\left(\frac{x}{N}\right) g^{2}\left(x-x^{\prime}\right) V\left(\frac{x^{\prime}}{N}\right) \underset{N}{\longrightarrow} c \int_{\mathbb{R}^{d}} V^{2}(y) d y .
$$

We have thus shown (4.7), and this concludes the proof of Lemma 4.1.

We will now conclude this short section with some remarks, in particular linking together (4.3), Lemma 4.1, and Theorem 3.3.

Remark 4.2. 1) By similar arguments as in Remark 3.4 we can view $\Phi^{N}$ as an $S^{\prime}\left(\mathbb{R}^{d}\right)$-valued random variable. The same proof as in Corollary 3.5 yields that

$$
\Phi^{N} \text { converges in law to } P^{G} \text {, as } N \rightarrow \infty \text {. }
$$

2) When

$$
u_{N} \frac{N^{d+2}}{b_{N}} \rightarrow \infty
$$

we can divide both members of (4.3) by $\sqrt{2 d u_{N}} N^{\frac{d}{2}+1}$. By Lemma 4.1 we then see that for each continuous compactly supported function $V$ on $\mathbb{R}^{d}$, both $\left\langle\widehat{\mathcal{L}}^{N}, V\right\rangle$ and $\left\langle\Phi^{N}, V\right\rangle$ converge in distribution to a centered Gaussian variable with variance $\langle V, G V\rangle$ (once again, note that $u_{N}$ enters the definition of $\widehat{\mathcal{L}}^{N}$ but not that of $\Phi^{N}$ ). One thus recovers the convergence asserted in Theorem 3.3 under the assumption

$$
\begin{cases}u_{N} N \rightarrow \infty, & \text { when } d=3, \\ u_{N} \frac{N^{2}}{\log N} \rightarrow \infty, & \text { when } d=4, \\ u_{N} N^{2} \rightarrow \infty, & \text { when } d \geq 5 .\end{cases}
$$

This recovers the full range of validity of Theorem 3.3 (i.e. $u_{N} N^{d-2} \rightarrow \infty$ ), when $d=3$, but only part of the range when $d \geq 4$. Observe that when $d \geq 4$, in 
the high intensity regime $u_{N} N^{d-2} \rightarrow \infty,\left\langle\widehat{\mathcal{L}}^{N}, V\right\rangle$ and $\left\langle\Phi^{N}, V\right\rangle$ have the same distributional limit, regardless of whether (4.14) breaks down or not.

3) One may wonder from the above discussion, whether some cancellations may be extracted from (4.3). In the high intensity regime $u_{N} N^{d-2} \rightarrow \infty$, given $M \geq 1$, can one for each $N$ couple two Gaussian free fields $\left(\varphi_{x}\right)_{x \in \mathbb{Z}^{d}},\left(\psi_{x}\right)_{x \in \mathbb{Z}^{d}}$, with $\left(L_{x, u_{N}}\right)_{x \in \mathbb{Z}^{d}}$, so that

$$
\begin{aligned}
\left(\varphi_{x}\right)_{x \in \mathbb{Z}^{d}} \text { is independent of }\left(L_{x, u_{N}}\right)_{x \in \mathbb{Z}^{d}}, & \\
\frac{1}{2} \varphi_{x}^{2}+L_{x, u_{N}}= & \frac{1}{2}\left(\psi_{x}+\sqrt{2 u_{N}}\right)^{2}, \text { a.s., for } x \in N C_{M} \\
& (\text { see (3.2) for notation), }
\end{aligned}
$$

and for any continuous function $V$ supported in $C_{M}$,

$$
\frac{1}{2} \sum_{x \in \mathbb{Z}^{d}} V\left(\frac{x}{N}\right)\left(\varphi_{x}^{2}-\psi_{x}^{2}\right) /\left(\sqrt{u_{N}} N^{\frac{d}{2}+1}\right) \underset{N}{\longrightarrow} 0 \text { in probability? }
$$

Such a coupling would yield a simple way to recover the identity of the distributional limits of $\left\langle\widehat{\mathcal{L}}^{N}, V\right\rangle$ and $\left\langle\Phi^{N}, V\right\rangle$ in the high intensity regime. Of course, when (4.14) holds such a coupling can be achieved (by (4.7) the last condition is automatically satisfied).

\section{The special case of dimension 3}

We have seen in the last section that when $d=3$ the isomorphism theorem (0.6) offers a quick route to the study of the asymptotic behaviour of $\widehat{\mathcal{L}}^{N}$ in the full range of the high intensity regime (0.4). In this section we investigate what happens "at the edge", in the constant intensity regime (0.2). The scaling limit of the distributional identity (0.6) (with proper counter terms) will bring as a byproduct an isomorphism theorem for 3-dimensional Brownian interlacements, see Theorem 5.1 and Corollary 5.3. This last corollary has a similar flavor to the distributional identity derived by Le Jan in the context of a Poisson gas of Brownian loops at half-integer intensity, see Chapter 10 §2, p. 104 of [23]. We recall the notation of the end of Section 1 concerning Gaussian free fields. Throughout this section $d=3$.

We denote by $H$ the Gaussian space, which is the $L^{2}\left(P^{G}\right)$-closure of

$$
\left\{\langle\Phi, f\rangle ; f \in S\left(\mathbb{R}^{d}\right)\right\} .
$$


For $Y, Z$ in $H$ the Wick product, see [18], p. 23, 24, :YZ: equals $Y Z-E^{P^{G}}[Y Z]$, and one has the identity, see [18], p. 11, 12 or [24], p. 201:

$$
\begin{aligned}
E^{P^{G}}\left[: Y^{2}:: Z^{2}:\right]= & 2 E^{P}[Y Z]^{2}, \text { for } Y, Z \in H \\
= & 2\langle f, G h\rangle^{2}, \text { when } Y=\langle\Phi, f\rangle, Z=\langle\Phi, h\rangle, \\
& \text { with } f, h \in S\left(\mathbb{R}^{d}\right)
\end{aligned}
$$

When $d=3$, the Green function $G\left(y, y^{\prime}\right)$ is locally square integrable, and for bounded measurable functions $V$ on $\mathbb{R}^{3}$ vanishing outside a compact set, one can define

$$
\int V(y): \Phi_{y}^{2}: d y=\lim _{\varepsilon \rightarrow 0} \int V(y): \Phi_{y, \varepsilon}^{2}: d y \text { in } L^{2}\left(P^{G}\right),
$$

where $\Phi_{y, \varepsilon}=\left\langle\Phi, \rho_{y, \varepsilon}\right\rangle$, with $\rho_{y, \varepsilon}(\cdot)=\frac{1}{\varepsilon^{3}} \rho\left(\frac{-y}{\varepsilon}\right)$, and $\rho(\cdot)$ a non-negative, smooth, compactly supported function on $\mathbb{R}^{3}$ such that $\int \rho(z) d z=1$. This fact uses (5.1), the crucial local square integrability of $G(\cdot)$, and similar arguments as in the proof of Proposition 8.5.1, p. 153 of [15], see also [23], p. 101. In addition, the limit object in the left-hand side of (5.1) does not depend on the specific choice of $\rho(\cdot)$. For convenience, we will assume that $\rho(\cdot)$ is radially symmetric and supported in $B(0,1)$.

For $V$ as above, one also defines (in a simpler fashion) the element of $H$ :

$$
\int V(y) \Phi_{y} d y=\lim _{\varepsilon \rightarrow 0} \int V(y) \Phi_{y, \varepsilon} d y \text { in } L^{2}\left(P^{G}\right) .
$$

We now assume that we are in the constant intensity regime (0.2), and for $V$ a continuous compactly supported function on $\mathbb{R}^{3}$, we rewrite (4.2) as the following identity in distribution under $P^{g} \otimes \overline{\mathbb{P}}$ :

$$
\frac{1}{2} \sum_{x \in \mathbb{Z}^{3}} V\left(\frac{x}{N}\right): \varphi_{x}^{2}:+d N^{2}\left\langle\mathcal{L}^{N}, V\right\rangle \stackrel{\text { law }}{=} \frac{1}{2} \sum_{x \in \mathbb{Z}^{3}} V\left(\frac{x}{N}\right):\left(\varphi_{x}+\sqrt{2 u_{N}}\right)^{2}:,
$$

where

$$
:\left(\varphi_{x}+\sqrt{2 u_{N}}\right)^{2}:=: \varphi_{x}^{2}:+2 \sqrt{2 u_{N}} \varphi_{x}+2 u_{N}
$$

and

$$
: \varphi_{x}^{2}:=\varphi_{x}^{2}-g(0)
$$

We already know that $\left\langle\mathcal{L}^{N}, V\right\rangle$ converges in distribution to $\left\langle\mathcal{L}_{\alpha}, V\right\rangle$ (see Theorem 3.2). The limit behavior of the other terms in (5.4) is described by 
Theorem 5.1. ( $d=3, V$ continuous with compact support $)$

$$
\begin{aligned}
& \text { For } \alpha \geq 0 \text { and } u_{N}=3 \alpha / N\left(=d \alpha / N^{d-2}\right) \text {, as } N \rightarrow \infty, \\
& \qquad \frac{1}{3 N^{2}} \sum_{x \in \mathbb{Z}^{3}} V\left(\frac{x}{N}\right):\left(\varphi_{x}+\sqrt{2 u_{N}}\right)^{2}: \\
& \text { converges in law to } \int V(y):\left(\Phi_{y}+\sqrt{2 \alpha}\right)^{2}: d y
\end{aligned}
$$

(the last term can be defined as

$$
\int V(y): \Phi_{y}^{2}: d y+2 \sqrt{\alpha} \int V(y) \Phi_{y} d y+2 \alpha \int V(y) d y,
$$

but see also (5.10) below).

Under $P^{G} \otimes \mathbb{P}$ one has the distributional identity

$$
\frac{1}{2} \int V(y): \Phi_{y}^{2}: d y+\left\langle\mathcal{L}_{\alpha}, V\right\rangle \stackrel{\text { law }}{=} \frac{1}{2} \int V(y):\left(\Phi_{y}+\sqrt{2 \alpha}\right)^{2}: d y .
$$

Proof. We first observe that (5.5), (5.4) (choosing $\alpha=0$ to handle the first term of (5.4)) and Theorem 3.2 readily imply (5.6). Thus, we only need to prove (5.5). This is a case of lattice field approximation (see chapter $9 \S 5$ and $\S 6$ of [15], also Chapter 8 of [33]). However, the fact that we consider the $d=3$ situation and massless free fields, makes the set-up a bit different. Since some care is needed, see for instance [15], p. 185-187, we sketch the proof for the reader's convenience. We define

$$
\varphi_{y, N}=\sqrt{\frac{N}{d}} \varphi_{N y}(\text { with } d=3), \text { for } y \in \mathbb{L}_{N},
$$

and note that the first term of (5.5) equals

$$
\frac{1}{N^{3}} \sum_{y \in \mathbb{L}_{N}} V(y):\left(\varphi_{y, N}+\sqrt{2 \alpha}\right)^{2}: .
$$

We now choose $\varepsilon(N)>0$ tending to 0 not too fast so that

$$
\varepsilon(N) \rightarrow 0 \text { and } N \varepsilon^{3}(N) \rightarrow \infty, \text { as } N \rightarrow \infty .
$$

As we explain below, the claim (5.5) will follow once we establish the following three facts:

$$
\begin{gathered}
\lim _{N} \| \int V(y):\left(\Phi_{y}+\sqrt{2 \alpha}\right)^{2}: d y \\
-\int V(y):\left(\Phi_{y, \varepsilon}+\sqrt{2 \alpha}\right)^{2}: d y \|_{L^{2}\left(P^{G}\right)}=0,
\end{gathered}
$$




$$
\begin{gathered}
\lim _{N} \| \int V(y):\left(\Phi_{y, \varepsilon}+\sqrt{2 \alpha}\right)^{2}: d y \\
-\frac{1}{N^{3}} \sum_{y \in \mathbb{L}_{N}} V(y):\left(\Phi_{y, \varepsilon}+\sqrt{2 \alpha}\right)^{2}: d y \|_{L^{1}\left(P^{G}\right)}=0,
\end{gathered}
$$

and for small real $z$, i.e. $|z| \leq r_{0}$,

$$
\begin{aligned}
\lim _{N}( & E^{P^{G}}\left[\exp \left\{\frac{z}{N^{3}} \sum_{y \in \mathbb{L}_{N}} V(y):\left(\Phi_{y, \varepsilon}+\sqrt{2 \alpha}\right)^{2}:\right\}\right] \\
- & \left.E^{P^{g}}\left[\exp \left\{\frac{z}{N^{3}} \sum_{y \in \mathbb{L}_{N}} V(y):\left(\varphi_{y, N}+\sqrt{2 \alpha}\right)^{2}:\right\}\right]\right)=0
\end{aligned}
$$

We first explain how these three facts yield a proof of (5.5). By Theorem 3.50, p. 39 of [18], we can replace $L^{1}\left(P^{G}\right)$ by $L^{2}\left(P^{G}\right)$ in (5.11). Together with (5.10) we find that

$$
\begin{gathered}
\lim _{N} \| \frac{1}{N^{3}} \sum_{y \in \mathbb{L}_{N}} V(y):\left(\Phi_{y, \varepsilon}+\sqrt{2 \alpha}\right)^{2}: \\
-\int V(y):\left(\Phi_{y}+\sqrt{2 \alpha}\right)^{2}: d y \|_{L^{2}\left(P^{G}\right)}=0 .
\end{gathered}
$$

By Theorem 6.7, p. 82 of [18], we thus see that for some $r_{1}>0$,

$$
\sup _{N \geq 1} E^{P^{G}}\left[\cosh \left(\frac{r_{1}}{N^{3}} \sum_{y \in \mathbb{L}_{N}} V(y):\left(\Phi_{y, \varepsilon}+\sqrt{2 \alpha}\right)^{2}:\right)\right]<\infty .
$$

By (5.12), we then find that for some $0<r_{2}<r_{1} \wedge r_{0}$,

$$
\sup _{N \geq 1} E^{P^{g}}\left[\cosh \left(\frac{r_{2}}{N^{3}} \sum_{y \in \mathbb{L}_{N}} V(y):\left(\varphi_{y, N}+\sqrt{2 \alpha}\right)^{2}:\right)\right]<\infty .
$$

Thus, by a similar argument as below (3.12), we see that the laws under $P^{g}$ of the random variables $\frac{1}{N^{3}} \sum_{y \in \mathbb{L}_{N}} V(y):\left(\varphi_{y, N}+\sqrt{2 \alpha}\right)^{2}$ : are tight, and, when along a subsequence $N_{k}$ they converge in distribution to a random variable $U$, then, for $|z|<r_{2}, e^{z U}$ is integrable and one has

$$
\begin{aligned}
E\left[e^{z U}\right] & =\lim _{N_{k}} E^{P^{g}}\left[\exp \left\{\frac{z}{N^{3}} \sum_{y \in \mathbb{L}_{N}} V(y):\left(\varphi_{y, N}+\sqrt{2 \alpha}\right)^{2}:\right\}\right] \\
\stackrel{(5.12)}{=} & \lim _{N_{k}} E^{P^{G}}\left[\exp \left\{\frac{z}{N^{3}} \sum_{y \in \mathbb{L}_{N}} V(y):\left(\Phi_{y, \varepsilon}+\sqrt{2 \alpha}\right)^{2}:\right\}\right] \\
& =E^{P^{G}}\left[\exp \left\{z \int V(y):\left(\Phi_{y}+\sqrt{2 \alpha}\right)^{2}: d y\right\}\right],
\end{aligned}
$$


using in the last step both the uniform integrability of the variables under the expectation in the second line when $|z|<r_{2}$ by (5.14), and (5.13) (see Theorem 5.4, p. 32 of [3]). By analyticity, the equality between the first and the last term of (5.16) extends to the strip $\left\{z \in \mathbb{C} ;|R e z|<r_{2}\right\}$. Hence, the random variable $U$ has same distribution as $\int V(y):\left(\Phi_{y}+\sqrt{2 \alpha}\right)^{2}: d y$, regardless of the extracted subsequence $N_{k}$, and (5.5) follows.

There remains to establish (5.10)-(5.12). The claim (5.10) is an immediate consequence of (5.2), (5.3) and the identity : $\left(\Phi_{y, \varepsilon}+\sqrt{2 \alpha}\right)^{2}:=: \Phi_{y, \varepsilon}^{2}:+$ $2 \sqrt{2 \alpha} \Phi_{y, \varepsilon}+2 \alpha$.

We then turn to (5.11) and simply explain why

$$
\lim _{N}\left\|\int V(y): \Phi_{y, \varepsilon}^{2}: d y-\frac{1}{N^{3}} \sum_{y \in \mathbb{L}_{N}} V(y): \Phi_{y, \varepsilon}^{2}:\right\|_{L^{1}\left(P^{G}\right)}=0 .
$$

The case of the linear term (where $2 \sqrt{2 \alpha} \Phi_{y, \varepsilon}$ replaces : $\Phi_{y, \varepsilon}^{2}:$ ) is simpler to handle, and the constant terms (after developing the square in (5.11)) cancel each other. The quantity under the limit in (5.17) is bounded above by $I_{1}+I_{2}$, where

$$
\begin{aligned}
I_{1}= & E^{P^{G}}\left[\left(\int h_{N}(y): \Phi_{y, \varepsilon}^{2}: d y\right)^{2}\right]^{\frac{1}{2}}, \text { with } \\
& h_{N}(y)=\sum_{z \in \mathbb{L}_{N}}(V(y)-V(z)) 1\left\{y \in z+\frac{1}{N}[0,1)^{3}\right\}, \\
I_{2}= & \sum_{z \in \mathbb{L}_{N}}|V(z)| E^{P^{G}}\left[\left|\int_{z+\frac{1}{N}[0,1)^{3}}: \Phi_{y, \varepsilon}^{2}:-: \Phi_{z, \varepsilon}^{2}: d y\right|\right] .
\end{aligned}
$$

By (5.1) we see that

$$
\begin{aligned}
& I_{1}^{2} \leq 2 \int\left|h_{N}(y)\right|\left|h_{N}(y)\right| G_{\varepsilon}^{2}\left(y-y^{\prime}\right) d y d y^{\prime}, \text { where } \\
& \begin{aligned}
G_{\varepsilon}(y) & =E^{P^{G}}\left[\Phi_{y, \varepsilon} \Phi_{0, \varepsilon}\right] \\
& =\int \rho_{\varepsilon}(z-y) G\left(z-z^{\prime}\right) \rho_{\varepsilon}\left(z^{\prime}\right) d z d z^{\prime}, \text { for } y \in \mathbb{R}^{3} .
\end{aligned}
\end{aligned}
$$

Note that $\rho_{\varepsilon}(\cdot)$ is spherically symmetric with support in $B(0, \varepsilon)$ and $G$ harmonic on $\mathbb{R}^{3} \backslash\{0\}$, so that

$$
\begin{aligned}
G_{\varepsilon}(y) & =G(y), \quad \text { when }|y|>2 \varepsilon \\
& \leq \frac{c}{\varepsilon}\|\rho\|_{\infty}, \quad \text { when }|y| \leq 2 \varepsilon,
\end{aligned}
$$


where in the last step we used that $\int_{\left|z-z^{\prime}\right| \leq r} G\left(z-z^{\prime}\right) d z=c r^{2}$, for $r \geq 0$, $z^{\prime} \in \mathbb{R}^{3}$.

We introduce a box $\Lambda=[-M, M)^{3}$, with $M \geq 1$ integer, such that

$\Lambda$ contains all points within $|\cdot|_{\infty}$-distance 1 from the support of $V$.

Note that $h_{N}$ vanishes outside $\Lambda$ (cf. (5.18)), and converges uniformly to 0 as $N \rightarrow \infty$. Moreover, by (5.22) and the local square integrability of $G^{2}$, we see that $\int_{\Lambda \times \Lambda} G_{\varepsilon}^{2}\left(y-y^{\prime}\right) d y d y \rightarrow \int_{\Lambda \times \Lambda} G^{2}\left(y-y^{\prime}\right) d y d y^{\prime}$, as $N \rightarrow \infty$. Hence, see $(5.20)$,

$$
I_{1} \rightarrow 0, \text { as } N \rightarrow \infty .
$$

We then control $I_{2}$. We first note that $N^{3}|\Lambda|$ bounds the number of boxes $z+$ $\frac{1}{N}[0,1)^{3}$ intersecting the support of $V$ (with $|\Lambda|$ the volume of $\Lambda$, and $z \in \mathbb{L}_{N}$ ). Hence,

$$
I_{2} \leq\|V\|_{\infty}|\Lambda| \sup _{y \in \frac{1}{N}[0,1)^{3}} E^{P^{G}}\left[\left|: \Phi_{y, \varepsilon}^{2}:-: \Phi_{0, \varepsilon}^{2}:\right|\right] .
$$

Moreover, for $y \in \mathbb{R}^{3}$ we have

$$
\begin{aligned}
& E^{P^{G}}\left[\left(: \Phi_{y, \varepsilon}^{2}:-: \Phi_{0, \varepsilon}^{2}:\right)^{2}\right] \\
= & E^{P^{G}}\left[: \Phi_{y, \varepsilon}^{2}:\right]+E^{P^{G}}\left[: \Phi_{0, \varepsilon}^{2}:\right]-2 E^{P^{G}}\left[: \Phi_{y, \varepsilon}^{2}:: \Phi_{0, \varepsilon}^{2}:\right] \\
\stackrel{(5.1)}{=} & 4\left(G_{\varepsilon}^{2}(0)-G_{\varepsilon}^{2}(y)\right) \stackrel{(5.22)}{\leq} \frac{c(\rho)}{\varepsilon}\left(G_{\varepsilon}(0)-G_{\varepsilon}(y)\right) \\
\leq & \frac{c(\rho)}{\varepsilon} \frac{|y|}{\varepsilon^{2}}\left(\text { since }\left\|\left|\nabla G_{\varepsilon}\right|\right\|_{L^{\infty}\left(\mathbb{R}^{3}\right)} \leq \frac{c(\rho)}{\varepsilon^{2}}\right) .
\end{aligned}
$$

We thus see that

$$
I_{2} \leq c(\rho)\|V\|_{\infty}|\Lambda|\left(N \varepsilon^{3}\right)^{-\frac{1}{2}} \underset{N}{\longrightarrow} 0 \text { (by (5.9)). }
$$

This complete the proof of (5.11). We now turn to (5.12).

By (5.22) and (1.2), (1.3), (1.6) we have for $y, y^{\prime} \in \mathbb{L}_{N}, N \geq 1$,

$$
G_{\varepsilon}\left(y-y^{\prime}\right) \leq c(\rho)\left(\left|y-y^{\prime}\right| \vee \frac{1}{N}\right)^{-1} \text { and } g_{N}\left(y, y^{\prime}\right) \leq c\left(\left|y-y^{\prime}\right| \vee \frac{1}{N}\right)^{-1} \text {. }
$$

We can thus make sure that for small real $z$,

$$
\begin{aligned}
& \sup _{N, y \in \mathbb{L}_{N}} \frac{|z|}{N^{3}} \sum_{y \in \mathbb{L}_{N}} G_{\varepsilon}\left(y-y^{\prime}\right)\left|V\left(y^{\prime}\right)\right| \leq \frac{1}{2}, \text { and } \\
& \sup _{N, y \in \mathbb{L}_{N}} \frac{|z|}{N^{3}} \sum_{y \in \mathbb{L}_{N}} g_{N}\left(y-y^{\prime}\right)\left|V\left(y^{\prime}\right)\right| \leq \frac{1}{2}
\end{aligned}
$$


(incidentally, by (3.6) and the continuity of $G|V|$, we also have $|z|\|G|V|\|_{L^{\infty}\left(\mathbb{R}^{3}\right)}$ $\left.\leq \frac{1}{2}\right)$.

If $\Lambda_{N}=\Lambda \cap \mathbb{L}_{N}$, the matrix $\left(G_{\varepsilon}\left(y-y^{\prime}\right)\right)_{y, y^{\prime} \in \Lambda_{N}}$ is positive definite. Indeed, by (5.21) it suffices to note that $\rho_{\varepsilon}(\cdot-y), y \in \Lambda_{N}$, are linearly independent (one easily sees by taking Fourier transforms and using an analytic continuation argument that any linear relation between these functions is trivial).

By Lemma 5.2.1 on p. 198 of [24], or Proposition 2.14 on p. 47 of [41], for $z$ as above,

$$
\begin{gathered}
E^{P^{G}}\left[\exp \left\{\frac{z}{2 N^{3}} \sum_{y \in \mathbb{L}_{N}} V(y):\left(\Phi_{y, \varepsilon}+\sqrt{2 \alpha}\right)^{2}:\right\}\right] \\
=\frac{e^{\alpha z\left\langle V,\left(I-z \widetilde{G}_{\varepsilon} V\right)^{-1} 1\right\rangle_{\mathbb{L}_{N}}}}{\left(\operatorname{det}_{2}\left(\left(I-z \widetilde{G}_{\varepsilon} V\right)_{\mid \Lambda_{N} \times \Lambda_{N}}\right)\right)^{\frac{1}{2}}},
\end{gathered}
$$

where $\operatorname{det}_{2}\left(\left(I-z \widetilde{G}_{\varepsilon} V\right)_{\mid \Lambda_{N} \times \Lambda_{N}}\right)$ denotes the regularized determinant of the finite matrix $K$, which is the restriction to

$$
\Lambda_{N} \times \Lambda_{N} \text { of }\left(\delta_{y, y^{\prime}}-z G_{\varepsilon}\left(y-y^{\prime}\right) \frac{V\left(y^{\prime}\right)}{N^{3}}\right)_{y, y^{\prime} \in \mathbb{L}_{N}},
$$

that is (det $K) e^{-\operatorname{Tr}(K-I)}$ (where $\operatorname{Tr}$ stands for the trace), see [34], p. 75. In addition, with some abuse of notation, in the term in the exponential in the right-hand side of (5.31), $\widetilde{G}_{\varepsilon}$ stands for the linear operator on functions on $\mathbb{L}_{N}$ defined by a similar formulas as (1.8), with $g_{N}\left(y, y^{\prime}\right)$ replaced by $G_{\varepsilon}\left(y-y^{\prime}\right)$, so that $\widetilde{G}_{\varepsilon} V$ operates on bounded functions on $\mathbb{L}_{N}$ and $I-z \widetilde{G}_{\varepsilon} V$ is invertible by (5.29). Also, compared to the above references, we used the identity following from the Neumann expansion of $\left(I-z \widetilde{G}_{\varepsilon} V\right)^{-1}$ :

$$
\left\langle V,\left(I-z \widetilde{G}_{\varepsilon} V\right)^{-1} 1\right\rangle_{\mathbb{L}_{N}}=\langle V, 1\rangle_{\mathbb{L}_{N}}+z\left\langle V,\left(I-z \widetilde{G}_{\varepsilon} V\right)^{-1} \widetilde{G}_{\varepsilon} V 1\right\rangle_{\mathbb{L}_{N}} .
$$

In a similar manner, with analogous notation, for $z$ as above, we have

$$
\begin{gathered}
E^{P^{g}}\left[\exp \left\{\frac{z}{2 N^{3}} \sum_{y \in \mathbb{L}_{N}} V(y):\left(\Phi_{y, N}+\sqrt{2 \alpha}\right)^{2}:\right\}\right] \\
=\frac{e^{\alpha z\left\langle V,\left(I-z G_{N} V\right)^{-1} 1\right\rangle_{\mathbb{L}_{N}}}}{\left(\operatorname{det}_{2}\left(\left(I-z G_{N} V\right)_{\mid \Lambda_{N} \times \Lambda_{N}}\right)\right)^{\frac{1}{2}}},
\end{gathered}
$$

By Proposition 3.1 and (5.30) we have for $z$ as above

$$
\lim _{N}\left\langle V,\left(I-z G_{N} V\right)^{-1} 1\right\rangle_{\mathbb{L}_{N}}=\left\langle V,(I-z G V)^{-1} 1\right\rangle .
$$


By an analogous argument as for the proof of Proposition 3.1 and (5.29) we have

$$
\lim _{N}\left\langle V,\left(I-z \widetilde{G}_{\varepsilon} V\right)^{-1} 1\right\rangle_{\mathbb{L}_{N}}=\left\langle V,(I-z G V)^{-1} 1\right\rangle .
$$

As we now explain, the claim (5.12) will now follow once we prove that

$$
\begin{aligned}
& \sup _{N} \sum_{y, y^{\prime} \in \Lambda_{N}}\left(G_{\varepsilon}\left(y, y^{\prime}\right)^{2}+g_{N}\left(y, y^{\prime}\right)^{2}\right) \frac{1}{N^{6}}<\infty, \\
& \lim _{N} \sum_{y, y^{\prime} \in \Lambda_{N}}\left(G_{\varepsilon}\left(y, y^{\prime}\right)-g_{N}\left(y, y^{\prime}\right)\right)^{2} \frac{1}{N^{6}}=0 .
\end{aligned}
$$

Indeed from Theorem 9.2, on p. 75 of [35], one knows that for any $n \times n$ matrices $A, B$ (and $\|\cdot\|_{2}$ denotes the Hilbert-Schmidt norm)

$$
\left|\operatorname{det}_{2}(I+A)-\operatorname{det}_{2}(I+B)\right| \leq\|A-B\|_{2} \exp \left\{c\left(\|A\|_{2}+\|B\|_{2}+1\right)^{2}\right\}
$$

(the constant $c$ does not depend on $n$ ).

Assuming (5.35), (5.36) one thus finds that for small $z$

$\lim _{N} \operatorname{det}_{2}\left(\left(I-z \widetilde{G}_{\varepsilon} V\right)_{\mid \Lambda_{N} \times \Lambda_{N}}\right)-\operatorname{det}_{2}\left(\left(I-z G_{N} V\right)_{\mid \Lambda_{N} \times \Lambda_{N}}\right)=0$, and

the regularized determinants in (5.38) each belong to $\left[\frac{1}{2}, \frac{3}{2}\right]$ for $N \geq 1$

(we use (5.37) with $B=0$ and $\operatorname{det}_{2}(I)=1$ for this last fact).

Combined with (5.33), (5.34) the claim (5.12) then follows.

There remains to prove (5.35) and (5.36). The bound (5.35) follows from (5.28) in a straightforward fashion, and we concentrate on (5.36). We pick $\gamma>0$. The sum in (5.36) equals $J_{1}+J_{2}$, where $J_{1}$ collect the terms where $\left|y-y^{\prime}\right|>\gamma$ and $I_{2}$ the terms where $\left|y-y^{\prime}\right| \leq \gamma$. When $N$ is large, $G_{\varepsilon}\left(y-y^{\prime}\right)=G\left(y-y^{\prime}\right)$ for all $\left|y-y^{\prime}\right|>\gamma$ (see (5.22)), $G\left(y-y^{\prime}\right)-g_{N}\left(y-y^{\prime}\right)$ converges uniformly to zero on this set by (1.7). We thus find that $\lim _{N} J_{1}=0$. As for the second term $J_{2}$, by $(5.28)$ we have

$$
\varlimsup_{N} J_{2} \leq \varlimsup_{N} \frac{c(\Lambda, \rho)}{N^{3}}\left(N^{2}+\sum_{z \in \mathbb{L}_{N}, 0<|z| \leq \gamma} \frac{1}{|z|^{2}}\right) \leq c^{\prime}(\Lambda, \rho) \gamma .
$$

Letting $\gamma$ tend to 0 , we obtain (5.36). This completes the proof of (5.12) and hence of Theorem 5.1. 
Remark 5.2. 1) Note that for $V$ continuous with compact support on $\mathbb{R}^{3}$ one has

$$
\begin{aligned}
\mathbb{E}\left[\left(\int V(y): \Phi_{y}^{2}: d y\right)^{2}\right] & =2 \int V(y) G^{2}\left(y-y^{\prime}\right) V\left(y^{\prime}\right) d y d y^{\prime} \\
& \leq c\|V\|_{L^{1}\left(\mathbb{R}^{d}\right)}\left(\|V\|_{L^{\infty}\left(\mathbb{R}^{d}\right)}+\|V\|_{L^{1}\left(\mathbb{R}^{3}\right)}\right),
\end{aligned}
$$

and a similar bound holds for

$$
\mathbb{E}\left[\left(\int\left(V(y) \Phi_{y} d y\right)^{2}\right]=\int V(y) G\left(y-y^{\prime}\right) V\left(y^{\prime}\right) d y d y^{\prime} .\right.
$$

We can thus extend the linear map sending $V$ to $\int V(y):\left(\Phi_{y}+\sqrt{2 \alpha}\right)^{2}: d y \in$ $L^{2}\left(P^{G}\right)$ by continuity to $S\left(\mathbb{R}^{3}\right)$ for any $\alpha \geq 0$. By the regularization theorem, see Theorem 2.3.2, p. 24 of [17], one can find a version denoted by : $(\Phi+\sqrt{2 \alpha})^{2}$ :, which is an $S^{\prime}\left(\mathbb{R}^{3}\right)$-valued random variable defined on the canonical space where $P^{G}$ is defined such that for any $V \in S\left(\mathbb{R}^{3}\right)$,

$$
P^{G} \text {-a.s., }\left\langle:(\Phi+\sqrt{2 \alpha})^{2}:, V\right\rangle=\int V(y):\left(\Phi_{y}+\sqrt{2 \alpha}\right)^{2}: d y .
$$

2) The intensity measure of $\mathcal{L}_{\alpha}$ equals $\alpha d y$ (see (2.39)), and by similar considerations as in Remark 3.4 we can also view $\mathcal{L}_{\alpha}$ (after a possible redefinition on a set of measure 0 ) as an $S^{\prime}\left(\mathbb{R}^{3}\right)$-valued random variable defined on $(\Omega, \mathcal{A}, \mathbb{P})$.

As a consequence of Theorem 5.1 we obtain an isomorphism theorem for the occupation measure of Brownian interlacements on $\mathbb{R}^{3}$, which arises as the scaling limit (with suitable counter terms) of (0.6):

Corollary 5.3. $(d=3, \alpha \geq 0)$

Under $P^{G} \otimes \mathbb{P}$ one has the identity in law on $S^{\prime}\left(\mathbb{R}^{3}\right)$ :

$$
\frac{1}{2}: \Phi^{2}:+\mathcal{L}_{\alpha} \stackrel{\text { law }}{=} \frac{1}{2}:(\Phi+\sqrt{2 \alpha})^{2}:
$$

Proof. By Remark 5.2 we can obtain the identity (5.6) for any $V \in S\left(\mathbb{R}^{3}\right)$. Hence under $P^{G} \otimes \mathbb{P}$, the random variables

$$
\left\langle\frac{1}{2}: \Phi^{2}:+\mathcal{L}_{\alpha}, V\right\rangle \text { and }\left\langle\frac{1}{2}:(\Phi+\sqrt{2 \alpha})^{2}:, V\right\rangle
$$

have the same distribution for each $V \in S\left(\mathbb{R}^{3}\right)$, and (5.41) easily follows. 
Remark 5.4. In the context of Brownian loops on $\mathbb{R}^{d}, 1 \leq d \leq 3$ (suitably killed when $d=1,2$ ), Le Jan, see p.104 of [23], showed that $\frac{1}{2}: \Phi^{2}$ : (or its corresponding object when $d=1,2$ ) has the same law as the renormalized occupation field of a Poisson cloud of Brownian loops with intensity parameter $\alpha=\frac{1}{2}$.

Observe that here, in (5.41), no renormalization on $\mathcal{L}_{\alpha}$ is needed (and $\alpha$ varies over $\mathbb{R}_{+}$). Only terms involving the free field need renormalization.

\section{References}

[1] D. Belius. Cover times in the discrete cylinder. Available at arXiv:1103.2079.

[2] D. Belius. Gumbel fluctuations for cover times in the discrete torus. To appear in "Probab. Theory Relat. Fields", also available at arXiv:1202.0190.

[3] P. Billingsley. Convergence of probability measures. Wiley, New York (1968).

[4] K. Burdzy. Multidimensional Brownian Excursions and Potential Theory. Wiley, New York (1987).

[5] J. Černý and S. Popov. On the internal distance in the interlacement set. Electron. J. Probab., 17(29) (2012), 1-25.

[6] J. Černý and A. Teixeira. From random walk trajectories to random interlacements. Ensaios Matemáticos, 23 (2012), 1-78.

[7] J. Černý, A. Teixeira and D. Windisch. Giant vacant component left by a random walk in a random d-regular graph. Ann. Inst. Henri Poincaré Probab. Stat., 47(4) (2011), 929-968.

[8] M. Cranston and T.R. McConnell. The lifetime of conditioned Brownian motion. Z. für Wahrsch. verw. Geb., 65(1) (1983), 1-11.

[9] A. Drewitz, B. Ráth and A. Sapozhnikov. Local percolative properties of the vacant set of random interlacements with small intensity. To appear in "Annales de l'Institut Henri Poincaré, Probabilités et Statistiques", also available at arXiv:1206.6635.

[10] R. Durrett. Brownian motion and martingales in analysis. Wadsworth, Belmont CA (1984).

[11] A. Dvoretzky, P. Erdös and S. Kakutani. Double points of paths of Brownian motion in n-space. Acta Sci. Math., Szeged, 12B (1950), 75-81.

[12] X. Fernique. Processus linéaires, processus généralisés. Ann. Inst. Fourier, Grenoble, 17(1) (1987), 1-92.

[13] I.M. Gel'fand and N.Ya. Vilenkin. Generalized Functions. Academic Press, New York and London (1964).

[14] R.K. Getoor. Splitting times and shift functionals. Z. Wahrscheinlichkeitstheorie verw. Gebiete, 47 (1979), 69-81. 
[15] J. Glimm and A. Jaffe. Quantum Physics. Springer, Berlin (1981).

[16] G.A. Hunt. Markoff chains and Martin boundaries. Illinois J. Math., 4 (1960), 313-340.

[17] K. Itô. Foundations of Stochastic Differential Equations in Infinite Dimensional Spaces. Soc. Indust. Appl. Math., Philadelphia (1984).

[18] S. Janson. Gaussian Hilbert Spaces. Cambridge University Press (1997).

[19] O. Kallenberg. Random measures. Academic Press, New York (1976).

[20] H. Lacoin and J. Tykesson. On the easiest way to connect $k$ points in the random interlacements process. Available at arXiv:1206.4216.

[21] G.F. Lawler. Intersections of random walks. Birkhäuser, Basel (1991).

[22] Y. Le Jan. Markov loops and renormalization. Ann. Probab., 38(3) (2010), 1280-1319.

[23] Y. Le Jan. Markov paths, loops and fields, volume 2026 of "Lecture Notes in Math". Ecole d'Eté de Probabilités de St. Flour, Springer, Berlin (2012).

[24] M.B. Marcus and J. Rosen. Markov processes, Gaussian processes, and local times. Cambridge University Press (2006).

[25] G. Matheron. Random Sets and Integral Geometry. Wiley, New York (1975).

[26] P.-A. Meyer. Théorème de continuité de P. Lévy sur les espaces nucléaires. Séminaire Bourbaki, 311 (1965/66), 509-522.

[27] S. Port and C. Stone. Brownian motion and classical Potential Theory. Academic Press, New York (1978).

[28] E.B. Procaccia and J. Tykesson. Geometry of the random interlacement. Electron. Commun. Probab., 16 (2011), 528-544.

[29] B. Ráth and A. Sapozhnikov. Connectivity properties of random interlacement and intersection of random walks. ALEA Lat. Am. J. Probab. Math. Stat., 9 (2012), 67-83.

[30] B. Ráth and A. Sapozhnikov. The effect of small quenched noise on connectivity properties of random interlacements. Electron. J. Probab., 8(4) (2013), 1-20.

[31] V. Sidoravicius and A.S. Sznitman. Percolation for the vacant set of random interlacements. Comm. Pure Appl. Math., 62(6) (2009), 831-858.

[32] M.L. Silverstein. Symmetric Markov Processes. Lecture Notes in Math., 426 (1974), Springer, Berlin.

[33] B. Simon. The $P(\phi)_{2}$ Euclidean (Quantum) field theory. Princeton University Press (1974).

[34] B. Simon. Functional Integration and Quantum Physics. Academic Press, New York (1979).

[35] B. Simon. Trace Ideals and Their Applications. Am. Math. Soc., Providence, second edition (2005). 
[36] A.S. Sznitman. Brownian motion, obstacles and random media. Springer, Berlin (1998).

[37] A.S. Sznitman. On the domination of random walk on a discrete cylinder by random interlacements. Electron. J. Probab., 14 (2009), 1670-1704.

[38] A.S. Sznitman. Vacant set of random interlacements and percolation. Ann. Math., 171 (2010), 2039-2087.

[39] A.S. Sznitman. Random interlacements and the Gaussian free field. Ann. Probab., 40(6) (2012), 2400-2438.

[40] A.S. Sznitman. An isomorphism theorem for random interlacements. Electron. Commun. Probab., 17(9) (2012), 1-9.

[41] A.S. Sznitman. Topics in occupation times and Gaussian free fields. Zurich Lectures in Advanced Mathematics, EMS, Zurich (2012).

[42] A. Teixeira. Interlacement percolation on transient weighted graphs. Electron. J. Probab., 14 (2009), 1604-1627.

[43] A. Teixeira. On the size of a finite vacant cluster of random interlacements with small intensity. Probab. Theory Relat. Fields, 150(3-4) (2011), 529-574.

[44] A. Teixeira and D. Windisch. On the fragmentation of a torus by random walk. Commun. Pure Appl. Math., 64(12) (2011), 1599-1646.

[45] M. Weil. Quasi-processus. Séminaire de Probabilités IV, Lecture Notes in Math., 124 (1970), 217-239, Springer, Berlin.

Alain-Sol Sznitman

Departement Mathematik

ETH-Zurich

CH-8092 Zürich

SWITZERLAND

E-mail: alain-sol.sznitman@math.ethz.ch 